"This is the peer reviewed version of the following article: [Computer Graphics Forum, 2018, 37 (6), pp. 325 - 342], which has been published in final form at [https://onlinelibrary.wiley.com/doi/abs/10.1111/cgf.13327] This article may be used for noncommercial purposes in accordance with Wiley Terms and Conditions for Self-Archiving 


\title{
STATE OF THE ART ON STYLIZED FABRICATION
}

\author{
BERND BICKEL $^{1}$, PAOLO CIGNONI ${ }^{2}$, LUIGI MALOMO ${ }^{2}$, NICO PIETRONI $^{2,3}$ \\ ${ }^{1}$ IST AUSTRIA $\quad{ }^{2}$ ISTI - CNR $\quad{ }^{3}$ UTS - SYDNEY
}

\begin{abstract}
Digital fabrication devices are powerful tools for creating tangible reproductions of 3D digital models. Most available printing technologies aim at producing an accurate copy of a tridimensional shape. However, fabrication technologies can also be used to create a stylistic representation of a digital shape. We refer to this class of methods as "stylized fabrication methods." These methods abstract geometric and physical features of a given shape to create an unconventional representation, to produce an optical illusion, or to devise a particular interaction with the fabricated model. In this state-of-the-art report, we classify and overview this broad and emerging class of approaches and also propose possible directions for future research.
\end{abstract}

\section{INTRODUCTION}

Industrial prototyping aims at creating tangible representations of objects that are usually modeled using Computer Aided Design tools. In the last few years, we have observed the rise of a novel class of digital fabrication methods which have significantly changed the workflow and accelerated the process of creating prototypes. Indeed, modern digital fabrication technologies make it possible to easily manufacture shapes with geometric complexity that is unattainable by other methods.

However, while, for example, classical 3D printing can perform extremely well in terms of reproduction accuracy, these techniques still have several restrictions. The workspace is usually small, the printing process is time consuming, and, to achieve an accurate reproduction, the input geometry has to satisfy both geometric and physical/structural constraints. Therefore, designing and mapping a virtual object to a printable representation that results in the desired physical artifact is a challenging problem.

The needs to overcome additive 3D printing limitations and find new creative and practical uses has led to radically new approaches for the fabrication of digital shapes. While many methods focus on as-accurate-as-possible reproduction, others trade accuracy for obtaining a particular style of a fabricated object. This is a common path in many artistic contexts, which has led to the successful experimentation with novel materials both in painting and in sculpting. The renouncing of (hyper-)realism in favor of style has led to new abstractions and put the emphasis on different aspects of artifacts. Similarly, it is possible to take advantage of computational techniques to create non-realistic representations of a given digital shape, with the main goal of enlivening the perception of the spectator. In computer graphics, nonphotorealistic rendering techniques are preferred to realistic ones in many application domains. This occurs because the style of a geometric representation can be an essential element to communicate a certain "shape mood" to the observer. We believe that some of these ideas can also be exploited in the computational fabrication community to create tangible representations of visual data.

Following these concepts, instead of fabricating an exact copy of the digital shape, it is also justifiable to compute a stylized artifact or even reproduce or interpret the intended shapes with a completely novel medium, such as light and shadow. We call these novel directions stylized fabrication techniques. Hence, the scope of these methods is to abstract the shape of a digital object and create an artistic, yet often practically useful, real-world illustration. In this sense, the performance cannot be simply quantified by the accuracy of the representation but rather by its potential of offering an original, appealing geometric performance. While practically such stylized representations may also be motivated in many cases by reducing costs and printing time or overcoming manufacturing limitations, they can be (and are) intentionally used by artists to design particular optical or stylistic effects.

Stylized fabrication takes great advantages from a broad spectrum of techniques proposed in computer graphics. As we will show in the next sections, many of these techniques require the shape to be decomposed into simpler or even low-dimensional elements while keeping the main geometric characteristics that make a shape unique. This means transforming raw geometric representations (polygonal meshes or point clouds) to more structured shape abstractions. Hence, geometry processing and shape analysis techniques are the key ingredients for achieving this goal. Other techniques exploit the physical properties of the fabrication material, attempting to anticipate its behavior to gather the desired physical effect. In this case, physically-based simulation and model reduction become fundamental. Similarly, 
ray tracing and material appearance models can be useful for reproducing the shading of a fabricated object and creating nice optical illusions. However, these approaches are not straightforward applications of state-of-the-art techniques; rather, each problem is usually related to a demanding design challenge with its own peculiarity. Additionally, it is necessary to consider the fabrication and physical constraints involved when trying to bring a virtual effect to the real world.

\section{CllassificAtion}

We may classify the techniques by considering the different phenomena that are abstracted, modified, or taken into account when generating a stylized physical representation:

Shapes: These methods modify three-dimensional shapes to a simpler, more essential, representation. This usually allows the usage of highly constrained materials and devices that are popular and inexpensive, such as paper, flat wooden lists, or iron wires. Moreover, this class of methods often does not require sophisticated fabrication devices. Due to the simpler fabrication process, these methods scale relatively well with the overall size of the reproduction.

Materials: This class of methods utilizes unconventional fabrication materials as the mean to obtain a particular effect. For example, soft materials, cloth, or threads are employed. To anticipate the appearance of the fabricated shape, the mechanical properties of the materials should be taken into account in the design process. This kind of approach can also be used for large-scale productions.

Lighting and Shadow: We can consider light and shadows as our fabrication domain and discuss techniques that, by fabricating objects with specific geometries and materials, are able to obtain complex shadows and lighting effects. Lighting can also be exploited to allow the fabrication of simple $2.5 \mathrm{D}$ objects that, just by shading, seem to be much more complex representations: this ideas has been exploited for centuries in basreliefs.

Decompositions: It is also possible to decompose the shape into a set of disjointed pieces to allow the user to interact and play with it. Differently from other decomposition techniques that focus, for instance, on overcoming additive manufacturing volume limitations and keeping the assembled final shape as robust as possible, these methods aim to create a 3D puzzle in which the assembly process is part of the game. These kinds of representations may require the use of 3D printing devices, as each piece may be unique. However, due to the simplicity of their geometries, the pieces can be mass produced using mold injection.

Printing the Unprintable: The abstract domains of math and art usually require the production of surfaces that cannot be manufactured in the real world, especially considering the restrictions imposed by digital fabrication techniques. Hence, such abstract representations can be simplified or redesigned to make their fabrication possible, retaining the aesthetic and visual feeling for illustrative purposes.

This classification will be used in the following sections to present the discussed fabrication techniques. Moreover, at the end of the report, in Table 1], we offer a further, finer classification, based on a few common characteristics of all the reviewed methods.

\section{SHAPES}

Shape representations and shape processing are core elements for computational fabrication. In the following, we will focus on methods that produce a simpler, more essential, stylized representation from a given input shape. Many of these methods are based on fabrication techniques beyond classical additive 3D printing. As we will show in this section, all these methods make use of geometry processing and shape analysis tools to abstract or simplify an input shape while keeping the peculiar features that describe the fabricated object. However, the solution strategies are often motivated by the underlying primitives used for approximating the shape, such as developable surface patches, flat interlocking patches, or rods, which we will also use for classifying these methods. Most of the produced models have a primarily illustrational or artistic purpose and rely on fabrication techniques that are based on cheap materials, such as paper and/or conventional fabrication methods.

3.1. Developable and Foldable Patches. A common fabrication technique for sculptures involves cutting, bending, folding, and gluing surface patches, with papercrafting being probably the most prominent example. Most of the methods for papercraft design aim at deriving the best way to map a 3D shape onto flat $2 \mathrm{D}$ pieces of paper. This problem has some similarities with the problem of deriving an optimal parametrization; however, papercraft design requires low or no distortion in the mapping process (as paper is mostly inextensible). 
One commercially available technique [Tam] consists of unfolding an input 3D mesh into a planar domain, eventually introducing cuts. The foldability, that is, the possibility of flattening the object introducing a little distortion, is guaranteed only if the input model is triangulated at a very low resolution. While this method is intuitive and simple to implement, the resulting objects inherit all the geometric artifacts due to the low resolution of the input mesh; this behavior is particularly evident in the case of smooth surfaces. To mitigate this approximation problem, Mitani et al. [MS04b] proposed a method to split the input mesh into a set of smooth, developable paper strips that can be easily assembled together (Figure $1 \mathrm{a}$ ). The technique uses least squares conformal maps [LPRM02] to derive an initial segmentation of the model surface. Inner boundary lines are then added inside to each segment to preserve concave or convex features. Triangular strips approximating the model surface are then generated from inner and inter-segment boundaries. Once unfolded, the different pieces correspond to the paper cut-outs that can be used to craft the paper model. The approach proposed by Shatz et al. [STL06] extended the previous methods to derive a more compact, structurally sound patch layout rather than simple developable strips (Figure 1 1 ). Since the cut layout is simpler, the gluing process is also simplified. This technique starts from a very fine segmentation of the mesh, and then it associates for each patch a parametric surface (either a plane or a conic surface) that defines the approximation error. Adjacent patches are iteratively merged until the approximation error remains below a certain threshold. The cut layout is improved during the process to facilitate the assembling procedure. Massarwi et al. [MGE07] improved the previous method $\theta$ f [STL06] by measuring the approximation error with the Hausdorff distance and using generalized cylinders fitted onto the input geometry (rather than restricting to planes and cones). This technique covers a wider range of cases and provides a better representation of the original object. The approach proposed by Killian et al. [KFC*08] is certainly capable of deriving the most complex shapes out of a single sheet of paper. In this approach, the shape is
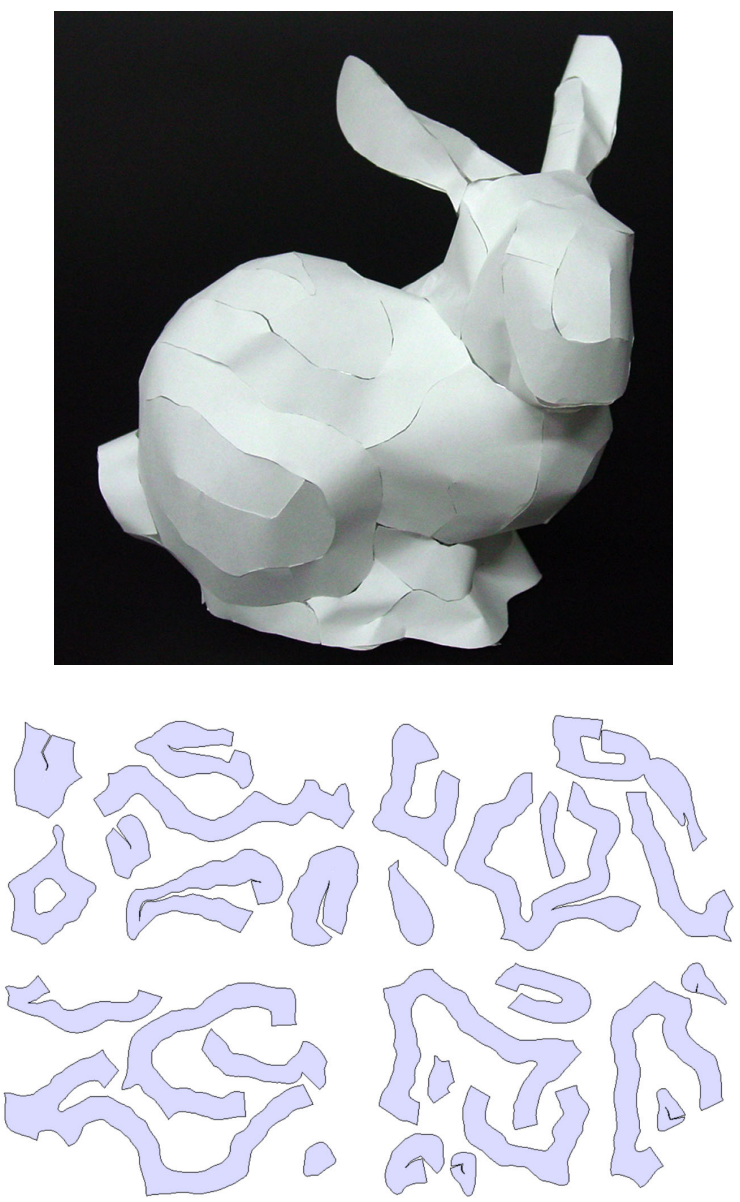

(a)
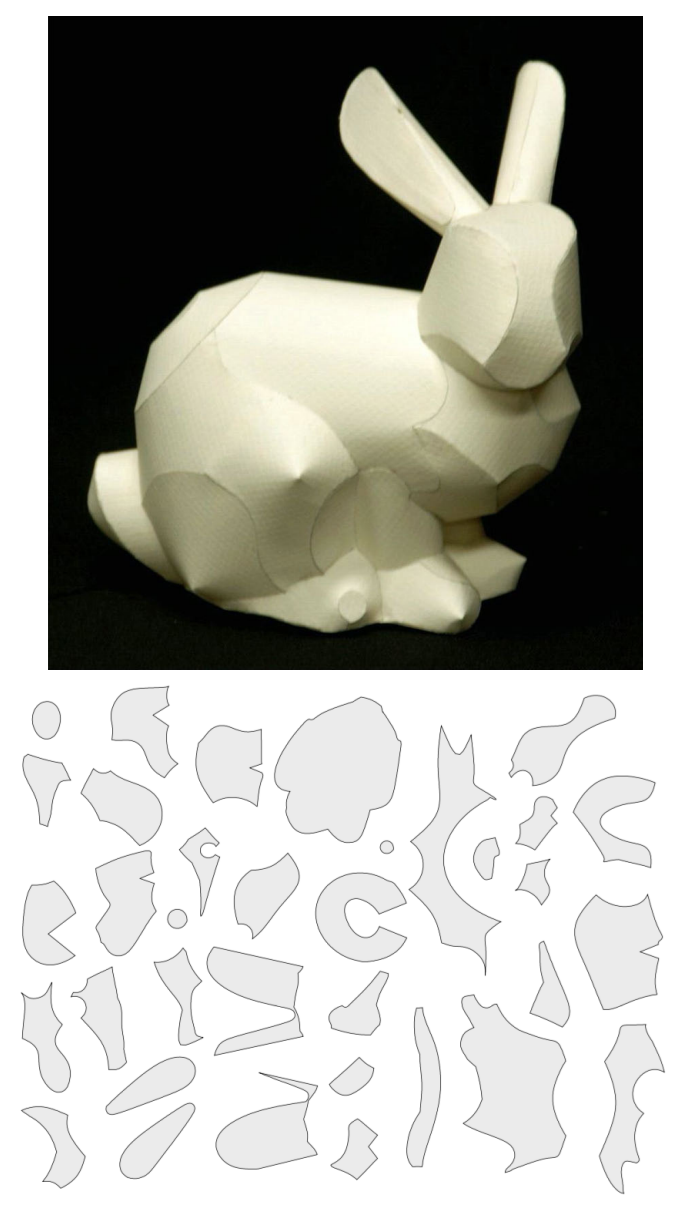

(b)

FIGURE 1. Assembled papercraft (top) and planar developable strips (bottom) produced with the approaches of (a) [MS04b] and (b) [STL06]. 


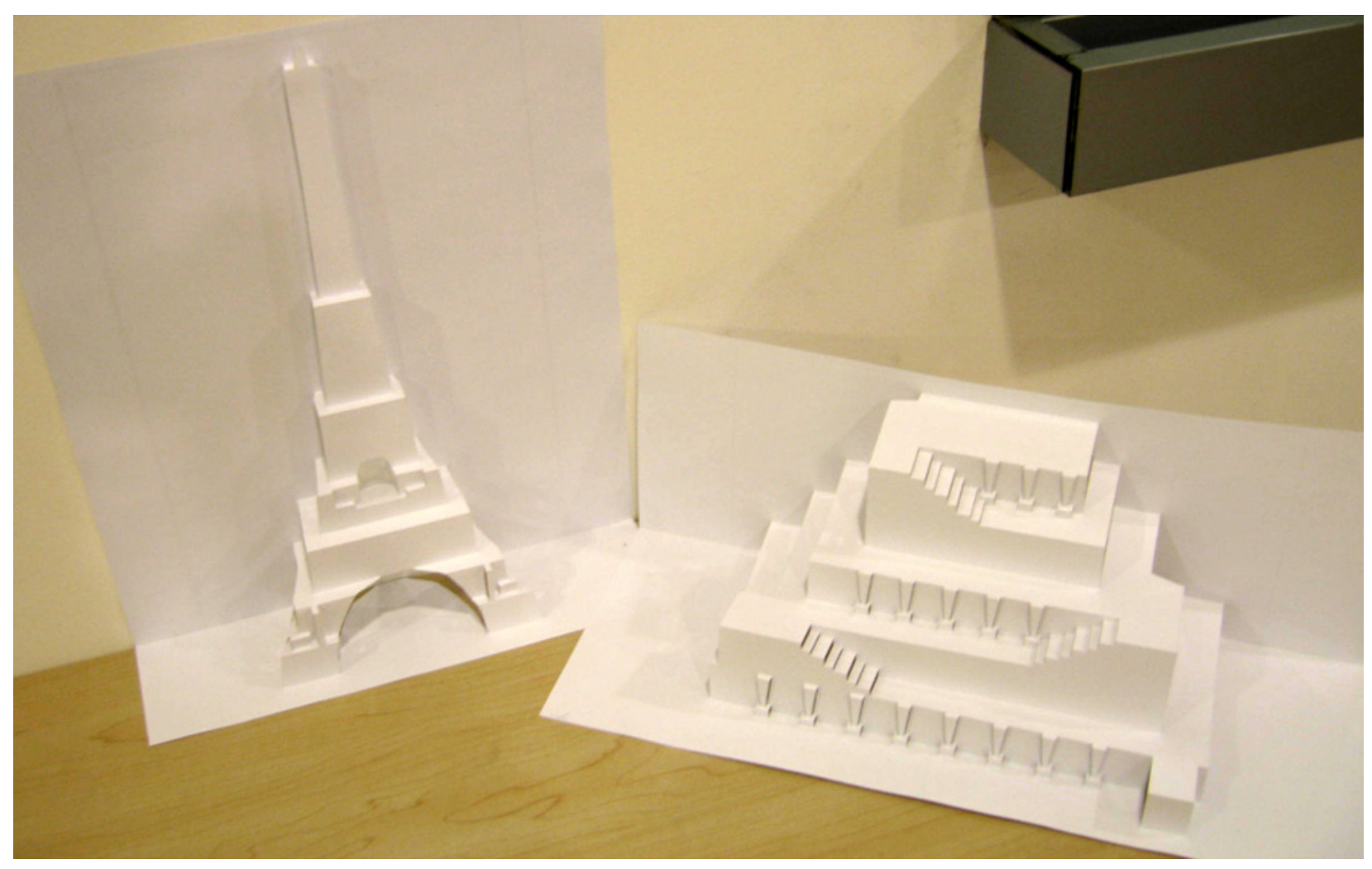

FIGURE 2. An example of origamic architecture generated with the technique in [[SSH*10].

decomposed into developable pieces with generic shapes. The method also uses curved folds and simultaneously optimizes the input shape and its planar developable representation. This technique can also be employed in architecture, as single-curvature developable surfaces are desirable when a shell structure must be covered with glass panels. Folding such patterns, however, is difficult, as multiple creases have to be folded simultaneously to obtain a properly folded target shape. Addressing this problem, Killian et al. [KMM17] introduced string-actuated, curved folded surfaces that can be shaped by pulling a network of strings. The concept of foldable strips has been extended by Takezawa et al. [TISM16] to manufacture complex freeform curves by interleaving two layers of orthogonal foldable strips aligned with principal curvature directions. Focusing on a different perspective, Zhang et al. [ZGPR16], instead of relying on automatic unfolding, propose the use of an interactive tool to decompose a 3D shape in simple pieces that can be easily fabricated by cardboard folding.

A radically different approach has been recently proposed by [AKW*16] and [TWZ*17], where the shape is decomposed in a set of developable strips of paper or flexible material that are arranged following the edges of an initial meshing.

When using paper as a base material and when the focus is on folding (instead of cutting and assembling), the main source of inspiration is origami. Origami is a papercrafting art that has been known for centuries. Traditionally, origami objects are manually designed; however, a generation of CG tools has been developed to automatize their design. Tachi [Tac10] proposed a novel method to create an origami craft depicting an input polyhedral 3D surface. Using tuck folding [Tac09], the method allows automatic reproduction of an input surface using a single-sheet folding pattern.

Conversely, other works take inspiration from paper pop-up illustrations. Pop-up illustrations are 3D figures that arise when a flat arrangement of paper sheets is opened. Pop-ups have two states: open (showing a 3D figure) and closed (reduced to a simple sheet of paper). Mitani and Suzuki [MS04a] proposed an interactive tool to design origamic architectures, a particular type of pop-up design made from a single sheet of paper, without any additional parts pasted on. This method produces a completely planar configuration of folds and cuts starting from a user-designed set of horizontal and vertical polygons that move to compose the target shape once the sheet is unfolded to 90 degrees. The technique shown in [ $\left.\mathrm{LSH}^{*} 10\right]$ improves the previous work by providing a method for automatically producing 


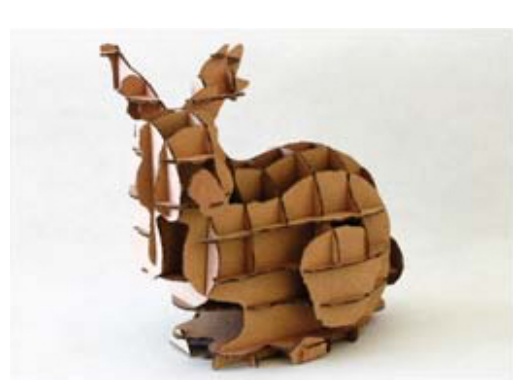

(a)

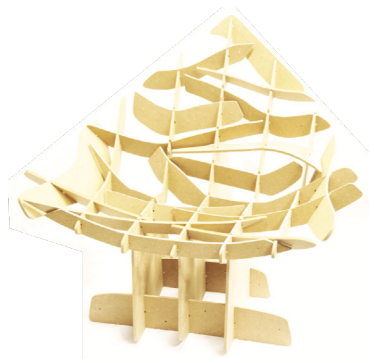

(b)

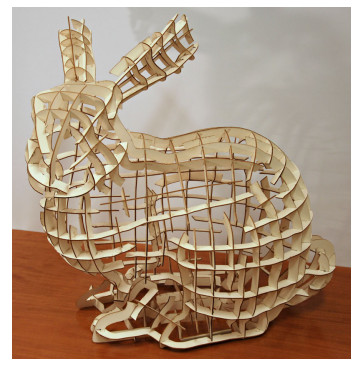

(c)

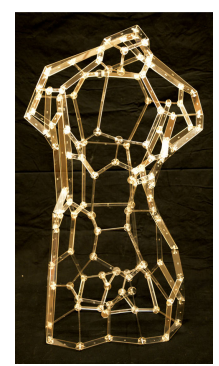

(d)

FIGURE 3. Interlocking planar slice designs obtained with (a) [HBA12], (b) [SP13], (c) [CPMS14], and (d) Beam Meshes [RA15].

an origamic architecture starting from an architectural 3D model. The output is a set of cuts and folds to be applied on a paper sheet. An approximated reproduction of the input model appears when the sheet is unfolded (Figure 2). Additionally, the algorithm guarantees that the pop-up model is stable, that is, the popping model has a unique unfolded configuration. The approach proposed by Le et al. [LLLN*14] also provides the automatic generation of origamic architectures but extends the range of possible shapes produced, providing a better approximation of the input model. $\mathrm{Li}$ et al. [LJGH11] tackle the problem of computer-assisted design and the automatic generation of the more generic V-Style pop-ups commonly found in children books. The method guarantees the feasibility of the produced pop-ups, which can be embedded into an actual book. This approach has recently been extended by Ruiz at al. [RLYL14] using a broader class of pop-up mechanisms.

3.2. Interlocking Flat Primitives. Planar slices can be easily manufactured using laser cutting and interlocked to create effective and efficient shape representations. Compared to traditional 3D printing, manufacturing planar cutouts is much cheaper and allows the use of many different material (e.g., cardboard, wood, acrylic, metal) in larger sizes. The usage of this technology for shape approximation has recently increased.

In [MSM11], the authors proposed an algorithm to create minimalistic representations of 3D objects using a limited set of planar 2D cross-sections. A user study was performed to understand how humans approximate a given object using cross-sectioning planes. The position of the planar slices has been correlated with relevant geometric features of the input shapes (ridges, valleys, symmetries, etc.), deriving an optimized weighting for each geometric feature. Combining a plane-space exploration and the above weights, a greedy iterative algorithm selects slices that capture as many important features as possible and converges to a set of planar proxies that approximate the object in a similar way as a user would do. In addition, the authors proposed to use the results to physically fabricate paper models using the obtained planar proxies as cut-outs, interlocked using colored guides as assembly hints. This interlocking slices approach has been fully developed by Hildebrand et al. [HBA12] for creating constructible sculptures made of cardboard cut-outs (Figure 3 a). The pieces are planar cross-sections of the input model that are interlocked together through a simple slit mechanism. The shape is decomposed using a modified BSP tree. The algorithm builds an approximated model by selecting and iteratively adding planar cut-outs with a specified inserting direction, which guarantees the feasibility of the insertion operation. For this purpose, the authors restricted the algorithm to use orthogonal or quasiorthogonal planes. This choice was made because the orthogonal slit interlocking mechanism provides the maximum grip between the planes. The final result consists of the sliding pieces and assembly instructions, which guarantee that the model approximation is constructible. This method has been extended by Schwartzburg and Pauly [SP13] to allow for generic configurations with non-orthogonal planes. The final configuration is designed to fulfill the requirements of fabrication, stability, and assembly [SP12]. Starting from a set of user-defined or automatically generated planar sections, the method automatically extracts from the input model the stencils for the resulting planar pieces and their insertion direction (Figure 3 3 ). During the optimization, it is ensured that two types of constraint are satisfied: (i) rigidity constraints, derived from the intersection angle and slit width of each interlock location, and (ii) slit constraints, guaranteeing that for each planar piece, an insertion direction and an ordering sequence exist to actually assemble them. A similar technique is presented in [CPMS14]: the method produces structures composed of shallow ribbon-shaped planar pieces that follow a cross field defined on the input mesh surface (Figure 36). These planar pieces interlock with each other using an extended slit mechanism. This enables non-orthogonal slice intersections and insertion movements that are not parallel to intersecting planes. Compared to the method in [SP13], this approach avoids the complexity of 

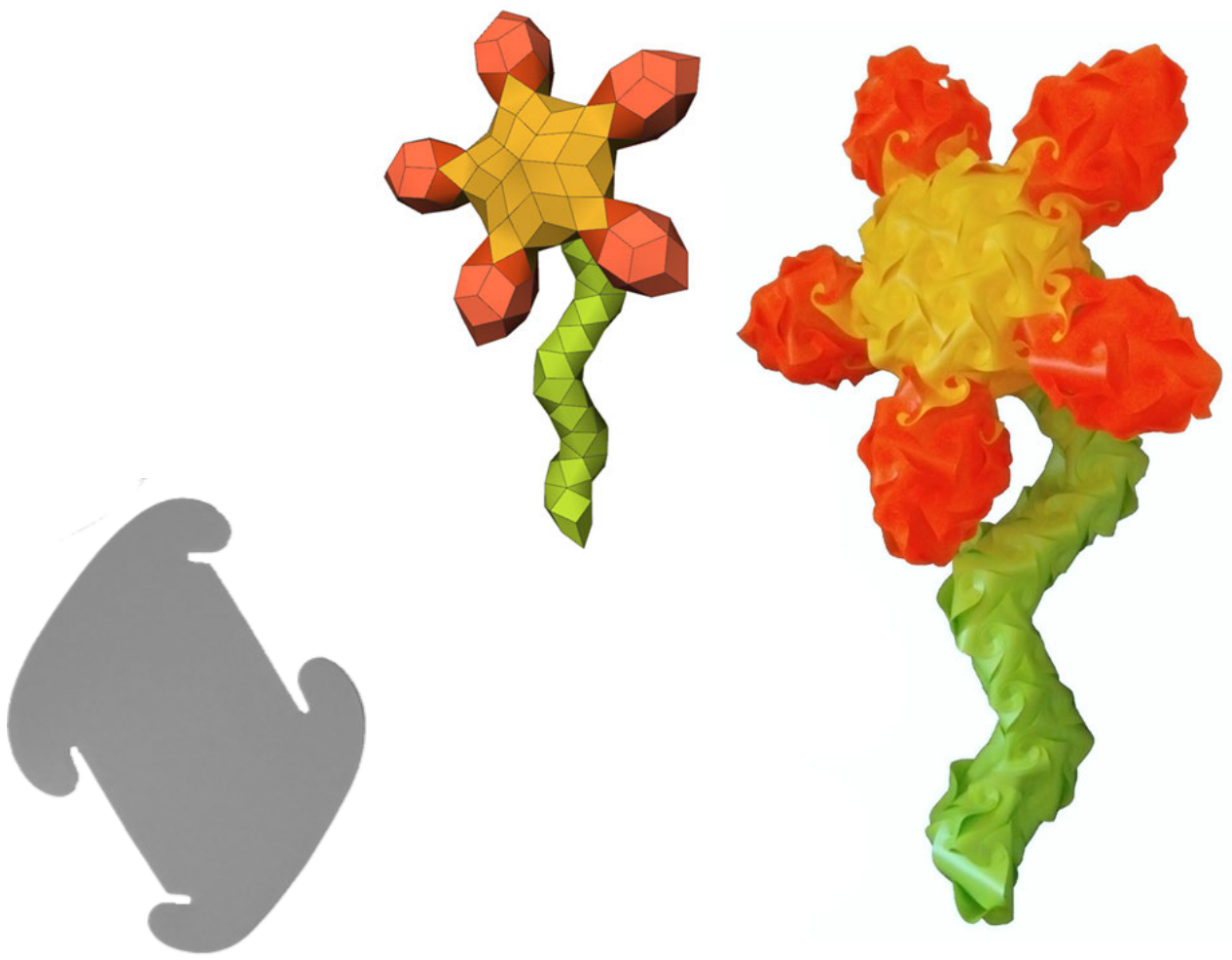

FIGURE 4. One example of a design obtained with the approach of [SCGT15] (right), composed by interlocking multiple elements (left).

considering multiple plane interlocks inside the object volume by using ribbon-shaped slices; as a byproduct, the final assembly is significantly simplified.

Beam meshes [RA15] approximate a 3D model with a set of interconnected beams with rectangular cross-sections (Figure 3 $\mathrm{d}$ ). The technique starts by approximating an input free-form mesh using a variant of the anisotropic centroidal voronoi tessellation; then, it uses the dual mesh to define an initial beam structure. Subsequently, local-global optimization is used to planarize each beam (torsion free). For fabrication purposes, planar joints are generated to hold collimating beam extremities, and, along with beam designs, they can be produced using laser cutting.

While the methods shown above offer automatic generation, FlatFitFab [MUS14] adopts an interactive approach. Specifically, it describes an interface to design objects composed of planar pieces that interlock orthogonally. Using an interactive interface, the user can design the main structures from scratch while the system provides physical feedback.

Chen et al. [CSaLM13] provided another interesting example of a fabrication technique to closely approximate an input 3D object. Given a target number of faces and an optional user-specified saliency map over the model surface, the method iteratively clusters mesh primitives to produce an arrangement of polygonal planar faces that approximate the input shape. The method ultimately produces a set of planar cut-outs that compose the approximated object, along with a series of (planar) joints that are used to connect them together. The output shapes can be fabricated with a laser cutter and then assembled. Moreover, each face of the resulting object can be easily textured according to the diffuse appearance of the original model. To provide color with the fabricated model, it is sufficient to print the colored cut-out and glue it on the external side of each corresponding piece.

Taking inspiration from IQlight lamps, the method proposed by Skouras et. al [SCGT15] produces suggestive objects made of flexible rhomboidal planar elements. These elements can be combinatorially interlocked to produce different configurations. The paper presented a tool to interactively design such objects: starting from one base shape (a configuration obtained from a limited set of elements), the tool allows the user to explore different options by modifying the object using two operations; extrusion, to select the extrusion profile among some base shapes, and merging, to combine distinct pieces by providing alternatives. The tool ultimately yields a design that allows the user to produce a surface by assembling homogeneous elements (Figure 4). 
3.3. Wires and Rods. Another class of techniques uses rigid rods or more generic flexible wires. This class of approaches gives a distinctive style to the objects. Unfortunately, wire meshes are in general very difficult to design manually. In this section, we briefly overview the computation tools that permit automatic or semi-automatic design.
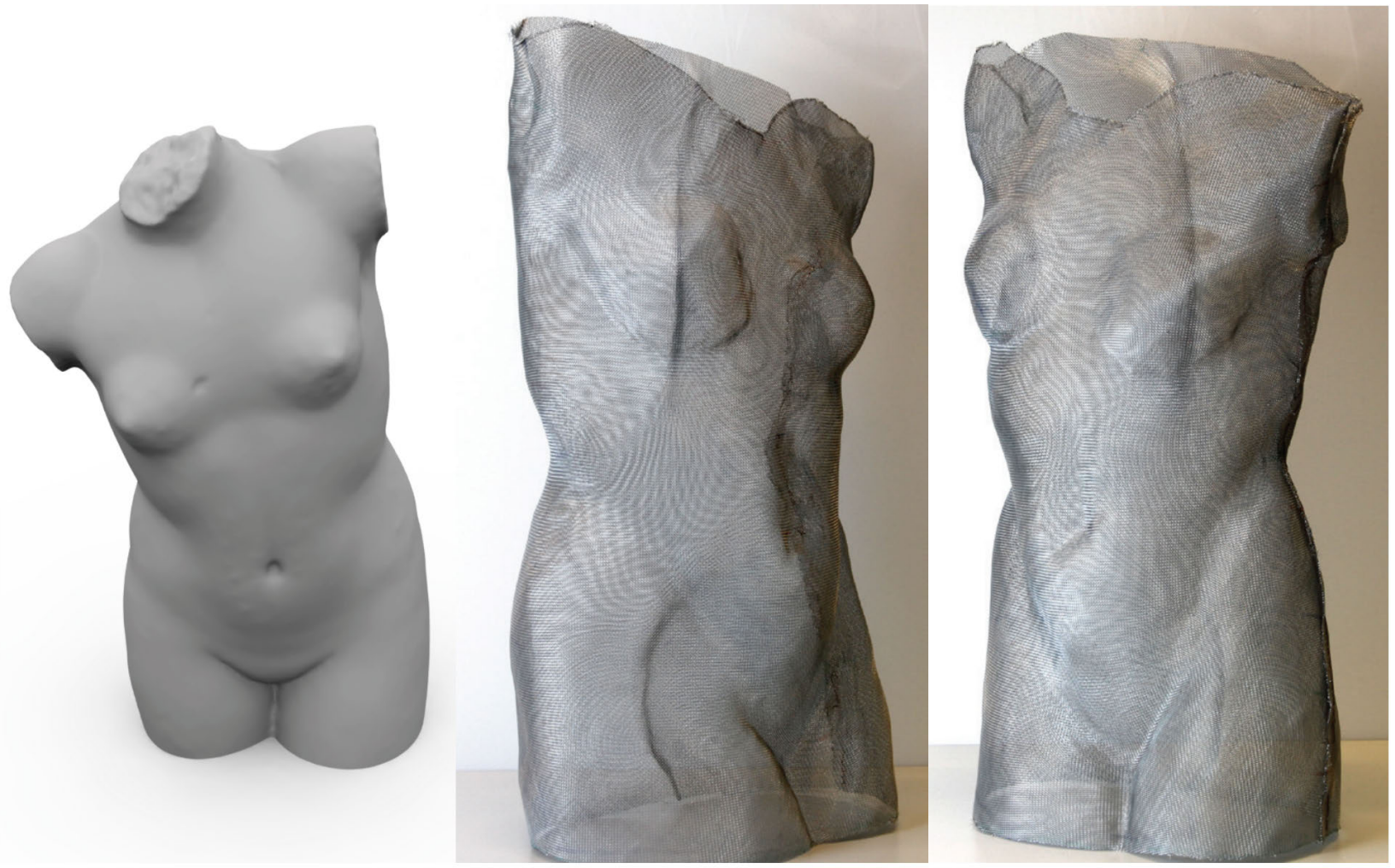

FIGURE 5. Fabricated wire mesh design [GSFD*14] (center and right) of a 3D model (left).

The approach in [GSFD*14] defines an interactive system to generate wire meshes (Figure 5). Such designs are composed by a single-sheet wire mesh (metallic woven wires arranged in a regular grid). This type of structure is quite popular among artists that usually employ a trial-and-error approach for creating their designs, by manually bending a piece of wire mesh to adapt it to a desired solid surface. The goal is to obtain a close reproduction of a target surface while satisfying the constraints of the wire sheet. The provided tool allows the user to place a set of constraints and define the regions where the wire mesh should better approximate the input geometry. A coarse to fine solver is used to simulate the effect of the wire mesh once it is attached to the solid object. As a result, a planar piece of wire mesh is produced with a corresponding approximate parametrization of the target 3D surface. For the actual fabrication, the wire sheet is attached, at prescribed locations, to a scaffold made of orthogonal interlocking pieces that capture the shape of the original surface (like the ones shown in Section 3.2.

Another use of wires is tailored to rapid prototyping. While originally $3 \mathrm{D}$ printing was conceived with this objective in mind, nowadays, the printing process is still relatively slow because printing medium-sized models can take tens of hours. The approach presented in [MIG*14] solves this problem by producing a wireframe preview for an input 3D model and generating printing instructions that are suitable for any FDM 3D printer. While this approach is created to speed up the object design process, it also offers an alternative to producing tangible illustrative representations that allow people to perceive the original object shape. The idea of wireframe printing has been extended in [HZH*16] and [WPGM16] using dedicated hardware combined with a robotic arm printer. To guarantee a proper printing, these algorithms optimize the movements of the arm, avoiding collisions during the printing process. The second approach has been extended in [PWMG16] to support interactive, on the fly printing during modeling. Yue et al. [YZY*17] propose to substitute the robotic arm with a user holding a cheap 3D extruder pen precisely driven by a mixed reality system.

Metal wires are also employed to produce jewelry artifacts. Iarussi et al. [ILB15] provided an interactive tool for designing wire-wrapped jewelry (Figure 6. The tool assists the user in addressing the two main challenges of 

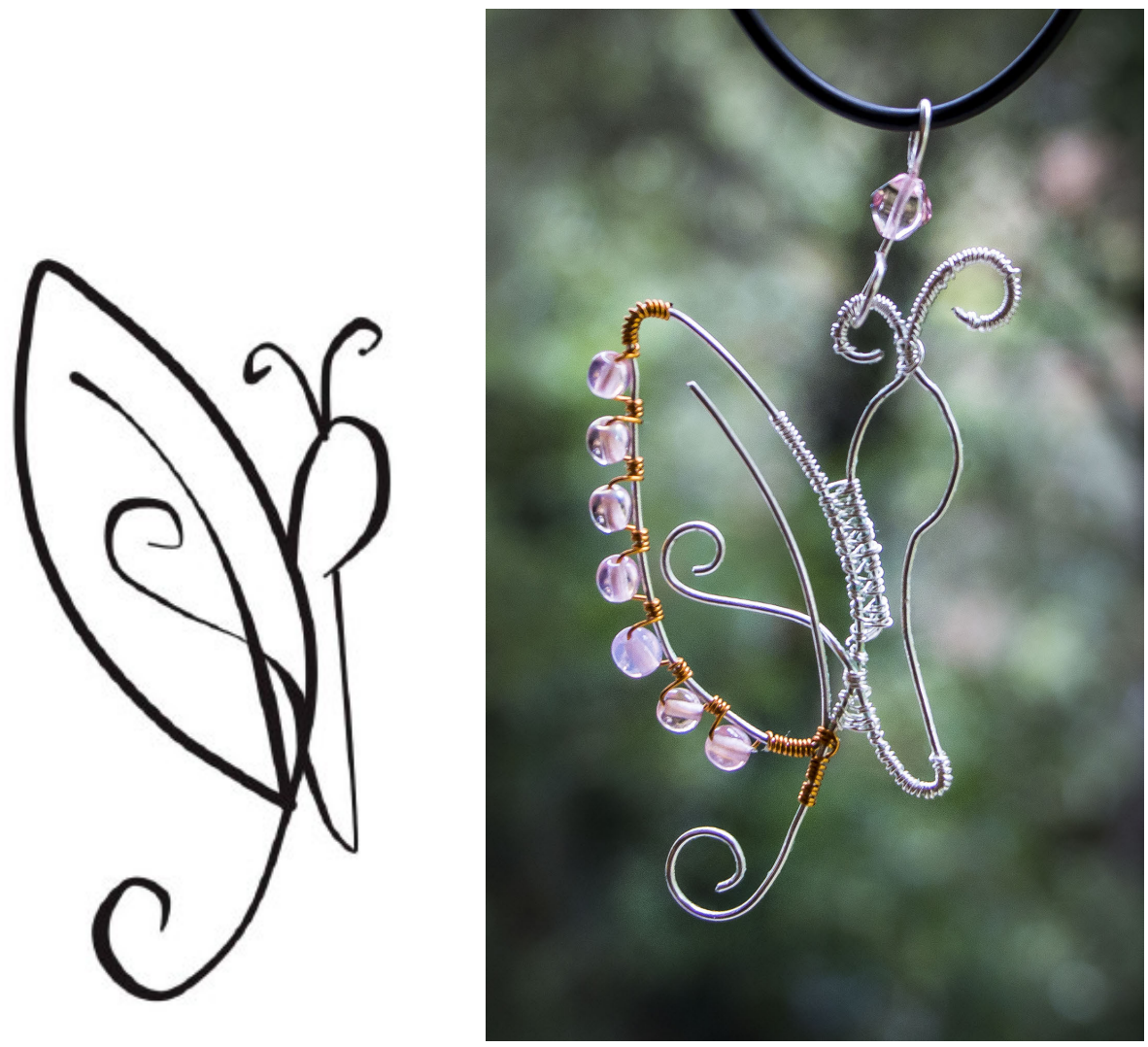

FIGURE 6. Starting from the 2D input image on the left, the approach in [ILB15] makes it possible to produce the design shown on the right.

designing such pieces: (a) decomposing an input drawing into a set of wires based on artistic and fabrication constraints and (b) generating an ad-hoc 3D-printable support structure to guide the manual wire-bending process. While the previous method is designed to produce 2D designs, the method of Miguel et al. [MLB16] creates 3D illustrative shape reproduction by the planar bending of metallic rods, achievable with a 2D wire-bending machine. Starting from an input 3D model and a set of planar cross-sectional contours, the system automatically computes a stable, selfsupporting wire sculpture. The output of the system provides instructions for a $2 \mathrm{D}$ wire-bending machine, and the obtained bent wires can be easily assembled without the need for connectors at contact points.

Combining solid objects and wires is also a very popular technique to produce small artifacts. In this context, Beady [IIM12] is a method that assists in the design and manufacturing of beadwork, which is the art of connecting beads together using wires. The design process of these objects is very difficult, and the assembly requires hours or even days. Beady offers an interactive tool to design such objects and also makes it possible to automatically produce a beadwork from an input 3D model. For the produced designs, the system offers step-by-step instructions to guide the wire path and beads insertion to ease the manual construction.

Other more sophisticated approaches include the use of texture synthesis [DLL*15], or the generation of procedural patterns [CZX*16,ZCT16] to synthesize visually appealing 1D patterns onto an input surface while keeping the overall structure structurally robust. The approach proposed by [MDLW15] optimizes a 2D piece for structural compliance under prescribed load conditions by using as little material as possible while trying to visually match a binary example pattern.

Recently, [YCC17] proposed a novel interactive method to design telescopic structures. Telescopic structures are composed of a set of nested shells that can be retracted and extended. The framework optimizes the curve of the telescopic structure such that each shell can be retracted or extended without colliding with neighboring shells.

Wire and rods can be used as structural elements carrying tension or compression to form tensegrity structures. Tensegritiy structures consists of a set of disjoint struts, tied together by cables connecting the endpoints of the struts 

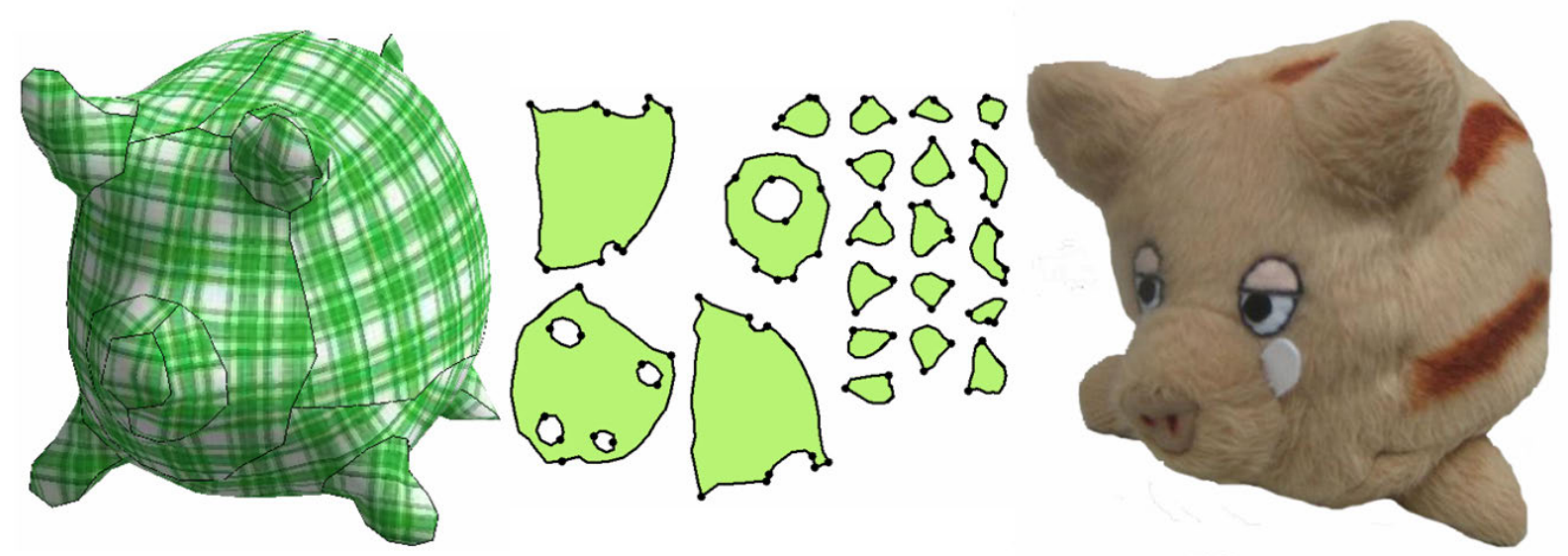

FIGURE 7. An example of plush Toy modelled with Plushie [MI07].

and nothing more. They behave as a stable structure balanced by structural elements carrying pre-tension (cables) or pre-compression (struts) forces. These structures are known for their desirable aesthetic and structural qualities, which are explored in architecture and art. Tensegrity structures can be modeled manually by assembling precomputed components [GCMT14] or automatically [Tac13], [TV $^{*}$ 17], optimizing both shape and tensions on elements to achieve stability.

\section{MATERials}

Besides its shape, the material is an inherent feature of an object. Recent developments in digital fabrication technologies have opened interesting avenues for reproducing solid objects with various material properties. With respect to the previous section, here the focus is on the behavior that the use of less conventional materials allows, for example, by considering soft materials [BBO* 10$]$. Even though the approaches proposed by Schumacher et al. [SBR*15], Panetta et al. [PZM*15], and Martinez et al. [MDL16] are beyond the scope of this report, they are worth mentioning because they make use of standard 3D printing technology to fabricate microstructures with desired elastic behaviors. The obtained objects are several orders of magnitude softer than the original material.

In general, soft materials allow for the production of objects with particular aesthetics and feel. In recent years, computer graphics research has led to the development of numerous tools that allow users to design soft objects, which can be classified by the lower-level primitives from which the object is assembled. Popular primitives are flat patches, which are sewn together; yarn, which is woven or knitted; or resin, which is either cast or 3D printed. Due to the distinct physical properties and fabrication constraints of these primitives, each of them results in specifically tailored design approaches.

Often, the mapping between low-level primitives and the final resemblance of the object is highly non-trivial. Addressing this challenge, Sensitive Couture, an interactive tool for garment design [UKIG11], allows users to manipulate the patch geometry in 2D and provides an interactive visualization of the draped dress in 3D. Similarly, Plushie [MI07] offers an interactive modeling system that supports users in designing their own original plush toys from scratch, providing a sketching interface for 3D modeling and editing (see Figure 7). Internally, the system constructs a 2D cloth pattern such that the simulation result matches the user's input stroke. Alternatively, assuming that the designer already has a target shape at hand, Pillow [MI06] presents a workflow for interactive flattening of patches starting from a given 3D shape. Inspired by this workflow, Skouras et al. [STK*14] presents an interactive optimization-in-the-loop tool for designing inflatable structures (see Figure 8). As the designer sketches the proposed placement of seams, the underlying optimizer supports the reverse-engineering of the physics of inflation, proposing a set of panels that best accommodate the desired seams and target shape. In all of these approaches, interactivity plays a key role in keeping the user in the loop for providing control over aesthetic considerations when determining a desired trade-off between 
the number and complexity of patches and the approximation quality of the 3D shape. Similarly, the problem of fabricating human-scale inflatable objects was explored in [SUS*17], also considering the dynamic aspects of the inflating process.

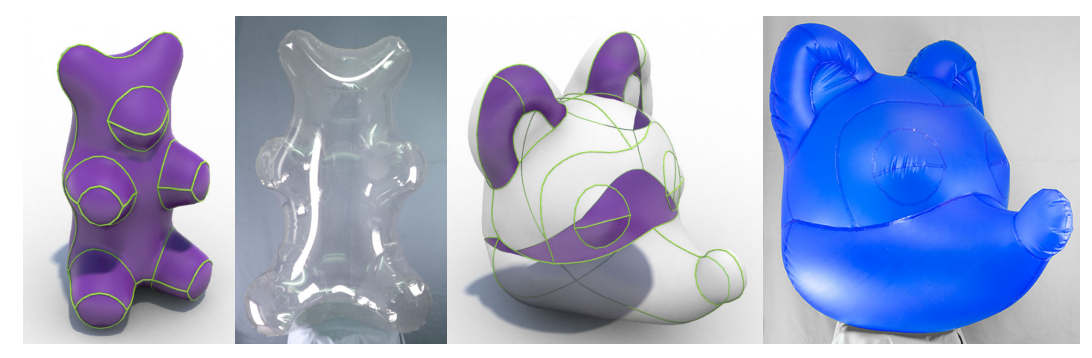

FIGURE 8. Examples of inflatable shapes produced by [STK*14], with the relative patch decompositions.

Alternatives to patch-based objects are inflatable membranes that can, for example, resemble complex shapes such as balloons [STBG12] or knit fabric, which does not need to be locally flat. While machine knitting is a mature technology, finding low-level machine instructions for non-trivial shapes requires a deep understanding of low-level knitting operations. To make this technology accessible to a wider audience, McCann et al. [MAN*16] proposed a compiler for 3D machine knitting that translates high-level shapes to machine instructions. In contrast, Hudson [Hud14] presented a new type of device that fabricates three-dimensional objects from soft fibers and demonstrated the process by manufacturing soft teddy bears.

Going beyond static objects, an active research area with growing interest involves self-actuating materials and selfshaping objects to generate 3D objects. Taking time into account as an additional dimension, the process of printing structures that can transform in a pre-programmed way in response to a stimulus is called 4D printing [Tib14]. Several recent works in computer graphics investigated the design of surfaces fabricated with pre-stretched elastic materials that deploy into complex, three-dimensional shapes [GMB17, POT17, KMM17]. Other methods optimize shapes to offer a predefined elastic [CLMK17] or dynamic behavior [UKSI14, BWBSH14]. Tailoring structured materials to obtain an unconventional material response is a largely unexplored but potentially highly interesting future research area for stylized fabrication.

\section{Lighting AND SHADOWS}

Beyond simpler shapes and materials with peculiar characteristics, we can consider light and shadows as our fabrication domain; we will discuss techniques that can either induce in the viewer the intuition of a richer 3D shape (as in bas-reliefs) or force light and shadows to assume precise complex shapes.

5.1. Low-Dimensional Representations of 3D Scenes. A low-relief (or bas-relief) can be seen as a way to exploit shading to obtain a physical representation of a 3D scene using only a thin layer of material; even by dropping out most of the geometric information the lighting of a 2.5D geometric approximation is able to convince the viewer of perceiving a complex 3D shape. Similarly, a high-relief also projects 3D geometry onto a thin layer of material; however, in this case, it keeps part of the original full 3D sculpting volume.

For these reasons, since antiquity, low- and high-reliefs have been widely used in the arts to approximate threedimensional shapes; to decorate cameos, sarcophagi, or architectural elements (e.g., Figure 9); or to create the engravings minted on coins.

Low-reliefs are a particularly reliable and lasting approach to representing a 3D shape onto an almost 2D space. From a fabrication point of view, low-reliefs significantly reduce the production costs since they can be fabricated using 2D milling subtractive techniques. Due to the reduced amount of material used, this reproduction technique scales very well to cover large surface areas. A survey on the specific problem of generating and modeling digital lowand high-reliefs can be found in [KWC*12].

Cignoni et al. [CMS97] proposed the first method for the automatic generation of a digital low-relief starting from a 3D model. The main issue in this class of problems is to find an efficient way to compress the depth range of an arbitrary 3D scene into the very limited thickness of a bas-relief. Several perceptive effects have been taken into account to solve this problem, such as the facts that there is a relation between the actual prominence of the shapes and their distance from the observer (more distant objects are usually flatter) and that the most important parts and details should be marked with higher reliefs. The approach proposed in [CMS97] used the perspective transformation 


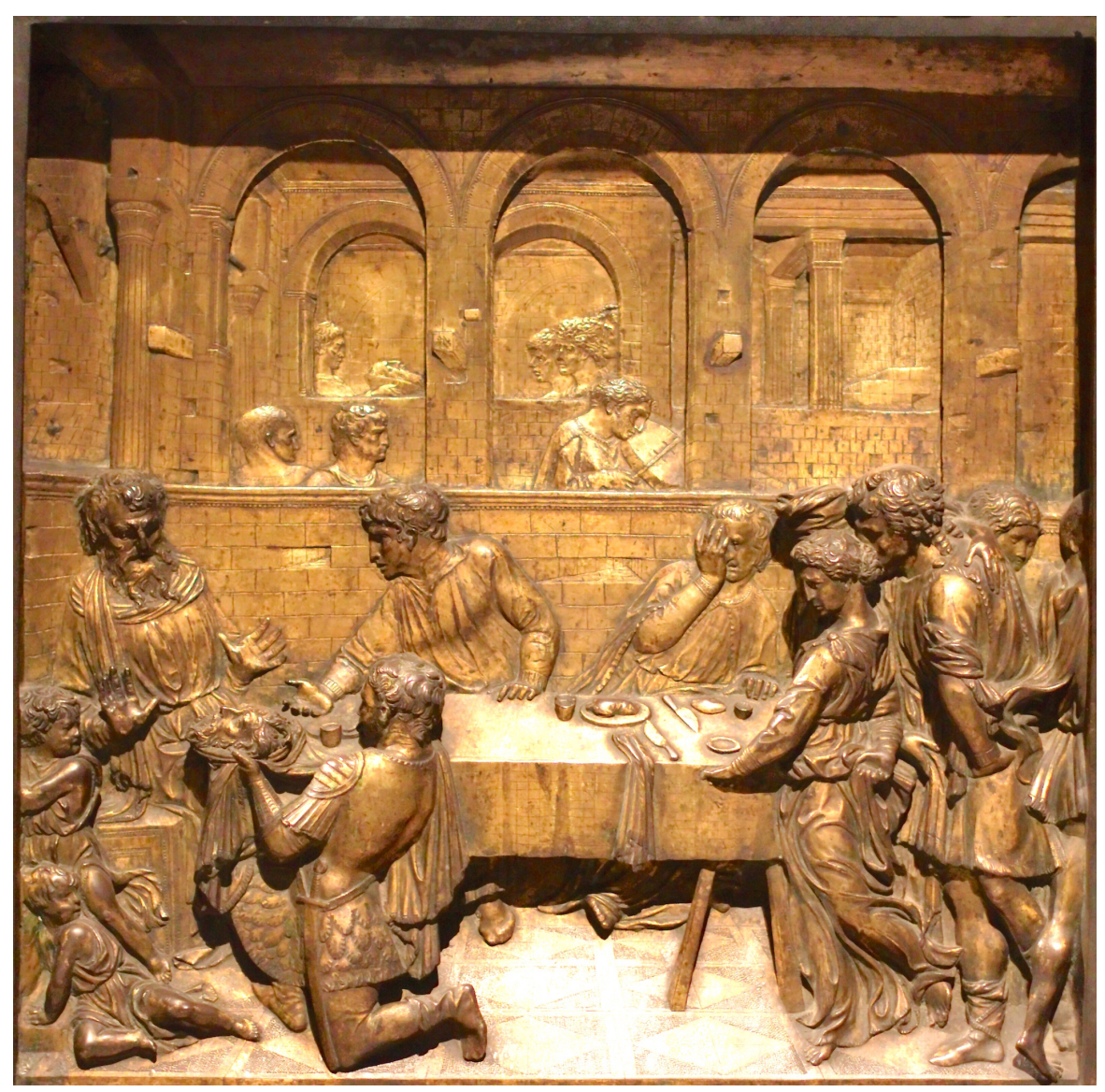

FIgURE 9. Herod's Banquet by Donatello (Siena, circa 1427), one of Donatello's earliest relief sculptures (photo by Matthias Kabel CC BY-SA 3.0 via Wikimedia Commons).

to compress the depth of the scene (i.e., using a factor $1 / p_{z}$, where $p_{z}$ is the depth of a point in the scene). While this method is able to reduce the elevation of more distant objects (see Figure 10), it cannot ensure the visibility of all the important details of the scene. This technique has been extended to use histogram equalization of the relief's depth map [SRML09] to distribute the height in the bas-relief in a more effective manner and thus preserve the details. Another class of methods for generating bas-reliefs [WDB*07, SBS07, KTB*09, BH11, ZZZY13, JMS14] achieves better results by considering that details are characterized by normal variations, and therefore they work in the gradient domain instead of using the depth values of the scene. In these approaches, the initial 3D model is used to generate a gradient field that is processed (eventually exploiting user input) to identify the most significant regions. The gradient field is finally integrated to recover the height field of the bas-relief. Other recent approaches [SPSH14, ASH15] have attempted to pose the problem as a more general deformation problem. They work directly on the input 3D mesh rather than a depth map. Such approaches are able to manage the problem of creating high-reliefs in a direct manner, as illustrated in Figure 11, where the objective is not the creation of a simple height field.

It is also worth noting that a bas-relief can be created starting directly from a simple 2D painting or a picture, translating it into a tactile 3D reproduction [RMP11|NR13], a task that has significant implications for visually impaired people, as it offers a direct way of accessing purely 2D information by means of a 3D medium.

A completely different approach is proposed by ShadowPix [BBAM12]. The technique is able to automatically create a height field capable of showing several images when illuminated from different (almost tangential) directions. Given a limited set of input images, an optimization procedure determines the proper height for each pixel such that, exploiting geometry self-shadowing, the field is able to closely approximate each image when illuminated from different directions.

5.2. Exploring Transparency. The recent advances in fabrication technologies make it possible to control, drive, and predict the reflectance behavior in a direct way. In a recent survey [ $\left.\mathrm{HIH}^{*} 13\right]$, all the specific aspects of the problem 


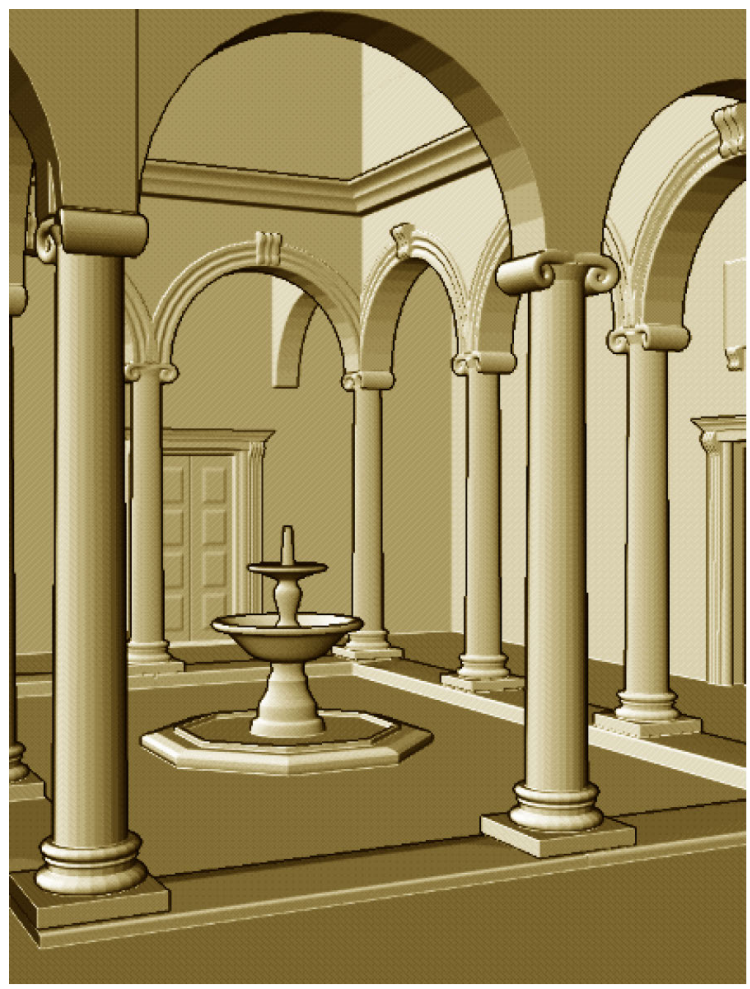

FIGURE 10. A bas-relief of a cloister generated with the approach proposed in [CMS97].

of creating physical workpieces with controllable appearance characteristics were discussed, including the relationship of this problem with display technologies. However, while most of these methods aim to replicate real-world effects, striving for accuracy, similar ideas can be used to produce nice stylized representations.

To control appearance, a basic approach consists of distributing materials with different refraction indices within the volume of an object. In [PRM14], the authors proposed the use of multi-material 3D printing to fabricate arbitrary surfaces with embedded optical fibers. The fibers are printed using two materials with different refraction indices: an external material with a low refraction index and an internal material with a higher one. The light propagates within the internal material and is trapped between the barriers provided by the external material. This makes it possible to use the internal reflections to guide the light to follow specific paths inside the volume of an object. In this way, the light can follow complex curved paths and transform an object surface into a custom shaped display (see Figure 12 a).

The method proposed by Yue et al. [YIC*12] exploits refraction to produce pixel art images. A flat panel is uniformly tessellated using a vocabulary of different sticks made of acrylate resin, with each stick refracting the light in a specific direction. An automatic optimization algorithm composes the panel such that a parallel light source is redirected in specific locations, composing the final a pixel art image on a flat surface. As shown by Figure $12 \mathrm{p}$, this approach enables the creation of panels that appear as opaque glass but produce complex patterns when traversed by light.

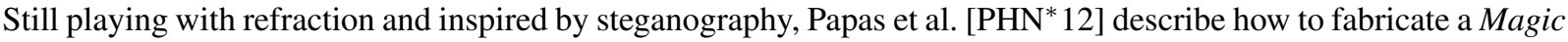
Lens that is capable of revealing multiple images when looking through it at a single pattern.

A completely different approach [HBLM11] creates multilayer models of an input 3D mesh. A multilayer model consists of a set of images embedded in glass panels that are stacked together (Figure 13). Each image displays the model at a different depth along the stack direction, and their composition results in a smooth 3D representation. The algorithm optimizes the images such that the multilayer model does not exhibit artifacts (self-shadowing or image splitting) when observed from multiple viewpoints.

5.3. Caustics Design. Another class of techniques aims to optimize the shape of a transparent object such that when it is hit by light, it paints a desired caustic image on a flat screen. The common idea among all these methods is that by slightly variating the normal of a surface, it is possible to re-direct light rays and concentrate them to compose a target image. Usually, the surface is fabricated using a milling machine. The approach proposed by Weyrich et al. [WPMR09] 


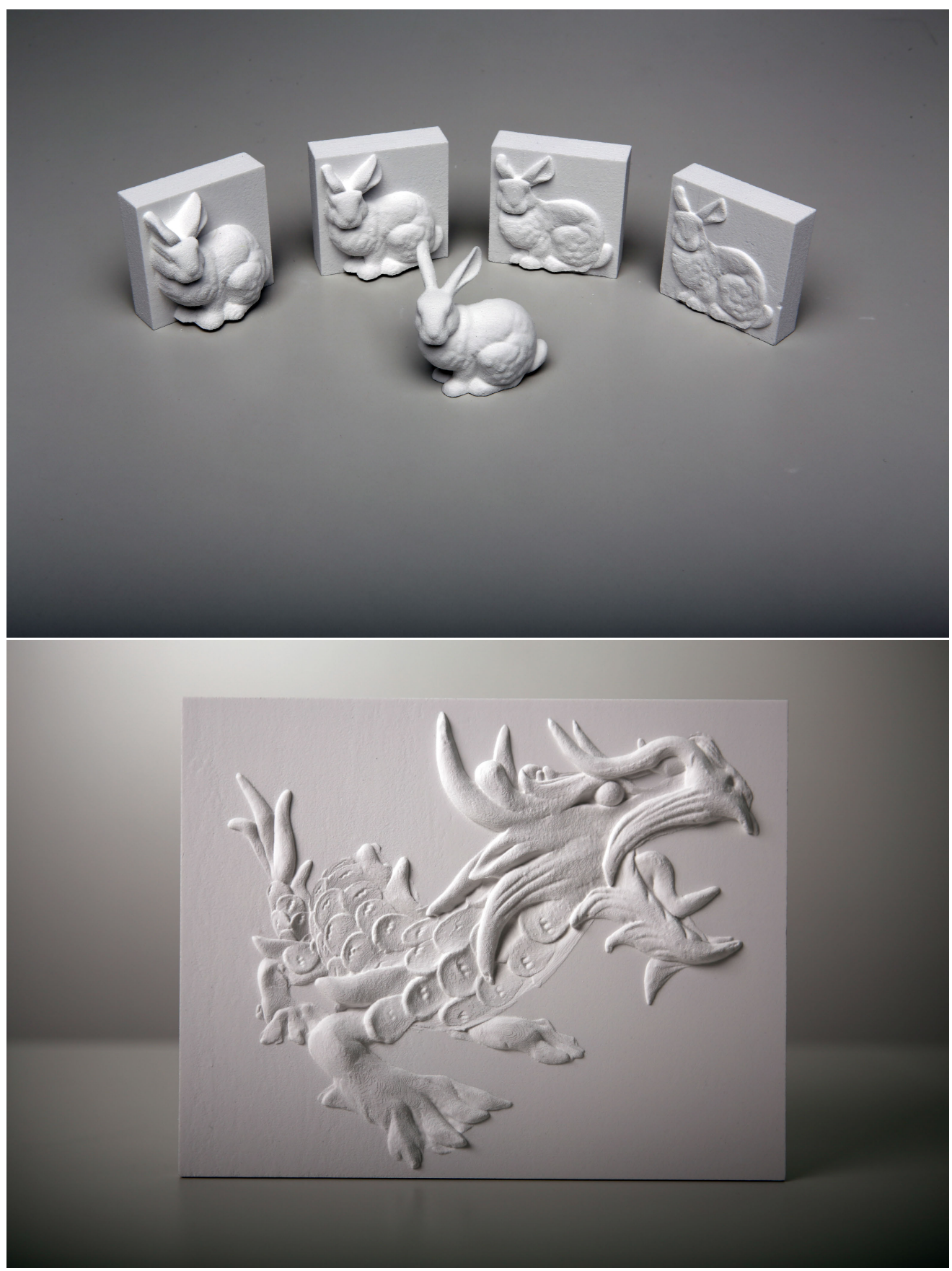

FIGURE 11. Two examples of high- and bas-reliefs automatically generated with the approach proposed in [SPSH14].

fabricates a set of reflective microfacets on a metallic surface using precision CNC milling. The objective is to deviate reflected light and concentrate it to match a target distribution specified by an input image. This approach has been extended by Papas et. al. [PJJ*11] using curved microfacets to reflect or refract light.

The approaches based on microfacets do not scale well when increasing the resolution of the target image and may create artifacts when trying to reproduce smooth features. These artifacts can be reduced by solving a global optimization problem [KEN*13] to retrieve a continuous mapping between the input light and the amount of photons that hit the target surface. In particular, in [YIC*14], the error in the target distribution is iteratively reduced following a globally smooth gradient field computed by solving a Poisson equation. The approach proposed by [STTP14] uses 

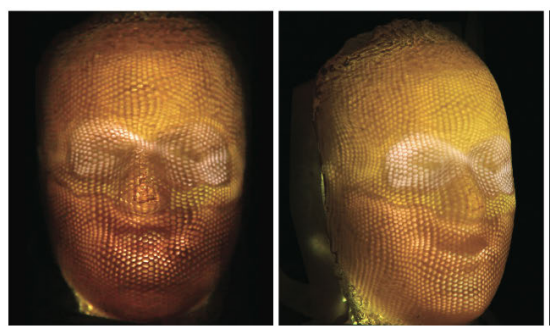

(a)
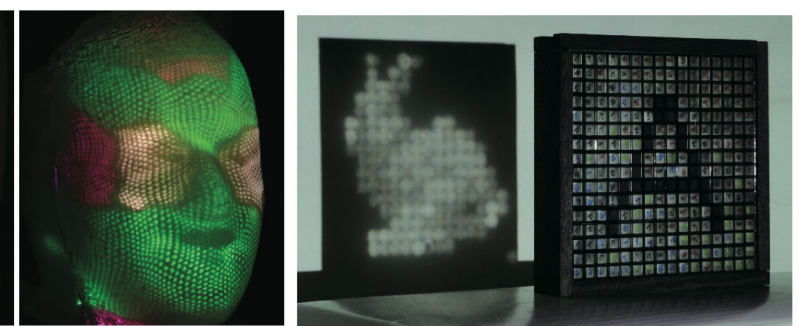

(b)

FIGURE 12. (a) A human face-shaped display fabricated by using the technique proposed in [PRM14]. (b) An example of a display fabricated using the approach of [YIC*12].
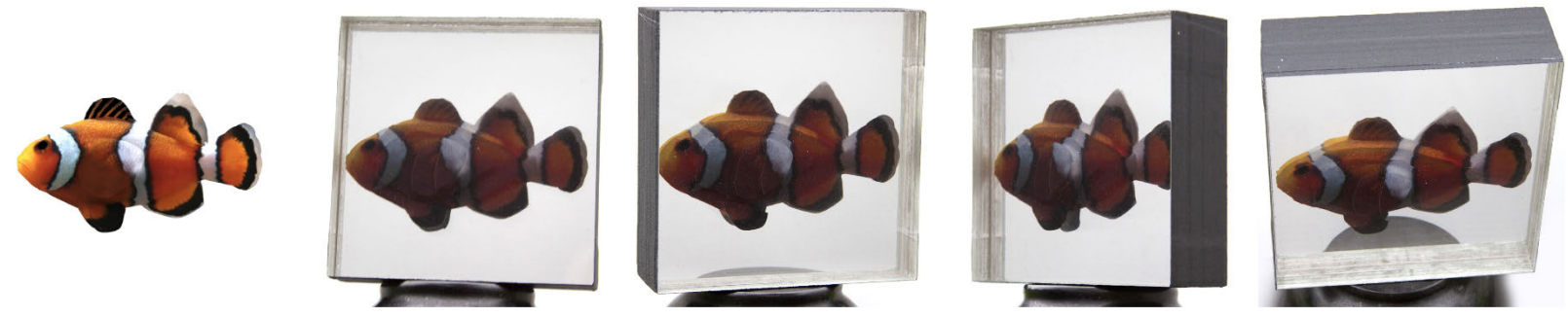

FIGURE 13. A multilayer model [HBLM11].
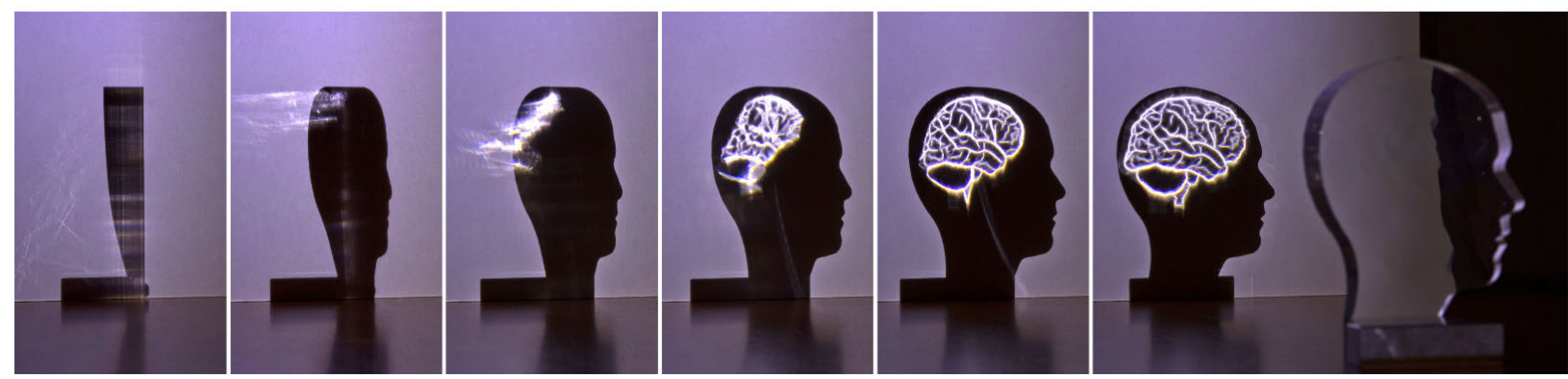

FIGURE 14. A transparent glass produced with the approach in [STTP14].

piecewise-smooth surfaces to allow the reproduction of sharp features. An example of caustics produced by this technique is shown in Figure 14.

5.4. Shadow Design. Shadows can be used as a medium to create suggestive effects. The approach proposed by Mitra and Pauly [MP09] creates 3D shadow art sculptures. The user selects a set of input images that represent several target shadow silhouettes. The system retrieves a single 3D shape that is capable of projecting all the silhouettes. Obviously, each silhouette appears when the object is illuminated from a specific direction. This effect is shown in Figure 15 This system uses a two-step optimization process; initially, it creates a shape by intersecting different volumetric shadow hulls and then slightly deforms the silhouettes to match the shadow constraint. The work of Zhao et al. [ZLW*16], instead, automatically generates 3D printable perforated lampshades that are able to project the luminance of a provided input image by modulating light and shadow. This technique accounts for the light emitted from a light bulb inside a static lampshade and optimizes the position and orientation of tubular holes on its surface, which control the light passage.

\section{Decompositions}

Several techniques focus on decomposing an object into multiple pieces. Many of the proposed methods aim to overcome the printing size limitations while keeping the produced shape structurally stable. However, another class 


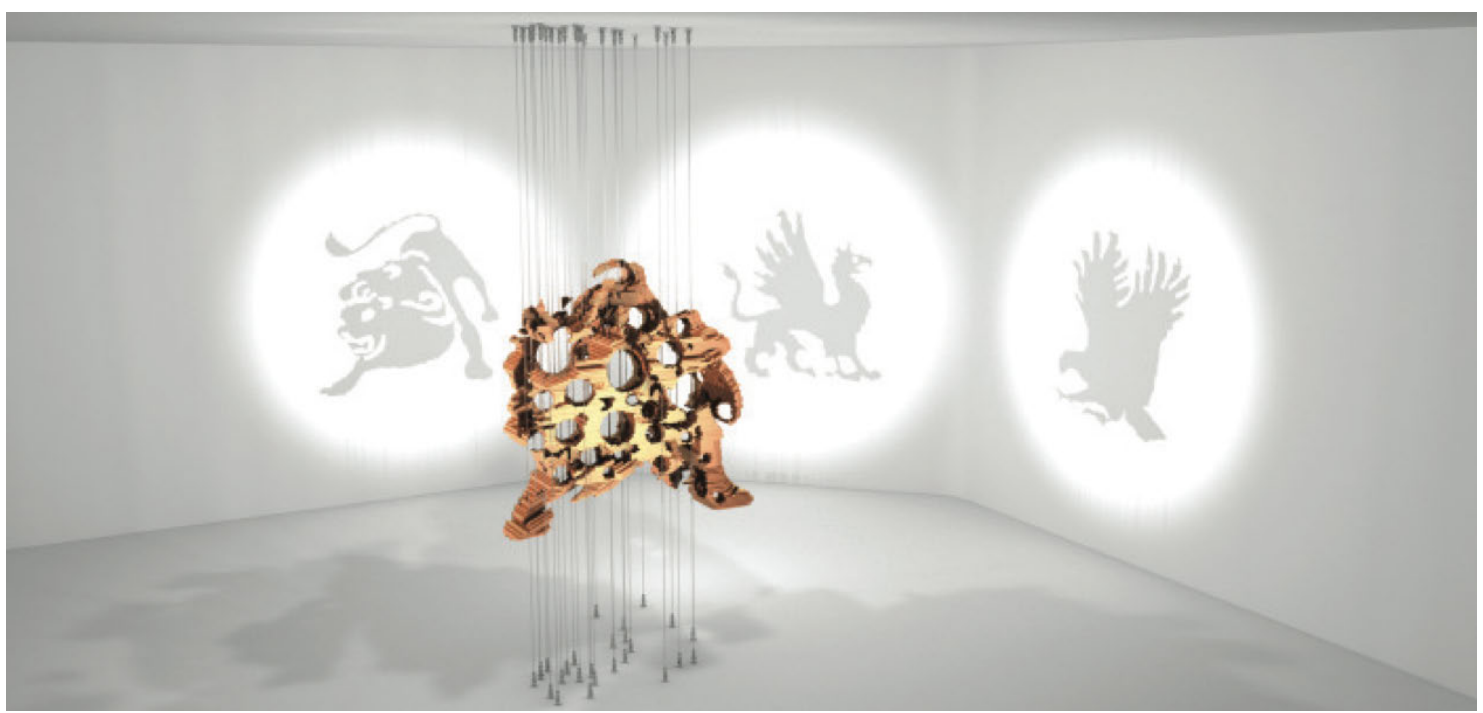

FIGURE 15. An example of shadow art design [MP09].

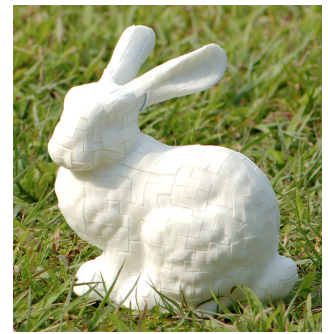

(a)

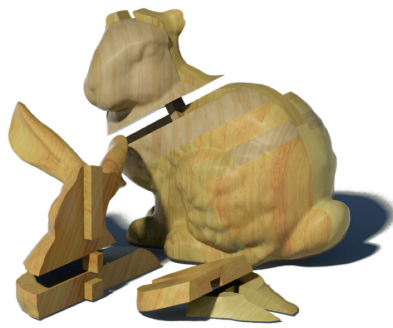

(b)

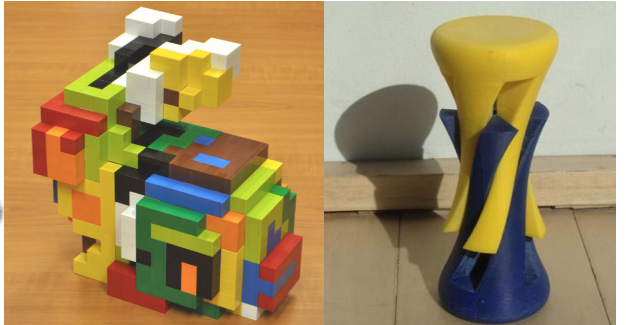

(c)

(d)

FIGURE 16. (a) 3D Polyomino puzzles [LFL09]; (b) Burr puzzles of [XLF*11]; (c) Recursive interlocking puzzles [SFCO12]; (d) Dissection puzzles [S1́2].

of methods focus on assemblable shapes. Some of these methods use a finite sets of pieces (e.g., LEGO blocks) and investigate how to obtain a stylized approximation of an input shape, while others aim to create a special assembly experience, similar to puzzles.

6.1. Generic Pieces. Some techniques investigate automatic 3D puzzle generation and place great attention on ensuring that a feasible assembling sequence exists. The 3D polyomino puzzle [LFL09] is an algorithm that generates interlocking 3D puzzles from a digital shape (see Figure 16 ). Given a quadrangulated mesh as input, the pieces are iteratively selected from a vocabulary and placed on the surface of the object until it is entirely covered. A valid assembly sequence is retrieved from the pieces graph and the partial ordering of inter-piece dependencies. While this method is limited to creating an external shell of the object, other methods decompose the entire volume. The burr puzzle technique [XLF*11] distributes a set of nodes inside a given 3D shape. Each node is used to create a mechanism that collects six volumetric pieces together, guaranteeing their mutual interlock (see Figure 16p). The approach proposed in [SFCO12] uses a more general placement of pieces. The object is split into a set of interlocking pieces made of grouped cubic voxels (see Figure 16). The peculiarity of this method is that it allows a unique assembly/disassembly sequence. In other words, for each step, it is possible to remove only a single piece at a time, as the others are locked together. As a byproduct, the resulting puzzle is kept stable during the whole assembly process. Dissection puzzles [S1́2] decompose simple input shapes into pieces that can be interlocked using complex movements such as rotations (rather than axis-aligned translations). The approach proposed in [SZ15] generalizes the idea of the Rubick's cube; the method decomposes an input model into a set of interlocking pieces, enabling manual reconfiguration of it along a set of finite configurations. 

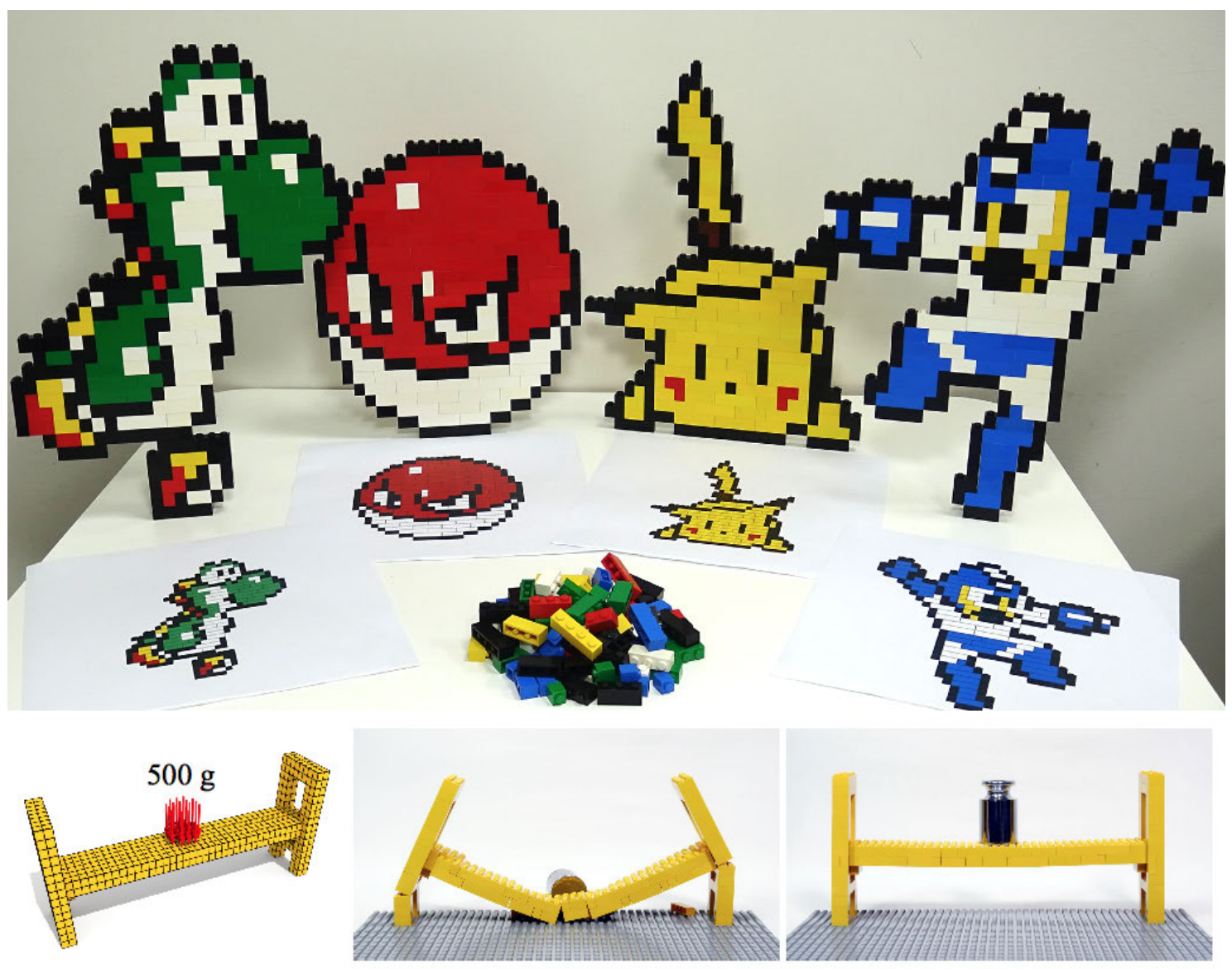

FIGURE 17. Top: an example of pixel-art modeled with LEGO blocks [KLC*15]; bottom: the approach proposed by [LYH*15] optimizes LEGO block compositions to support external loads.
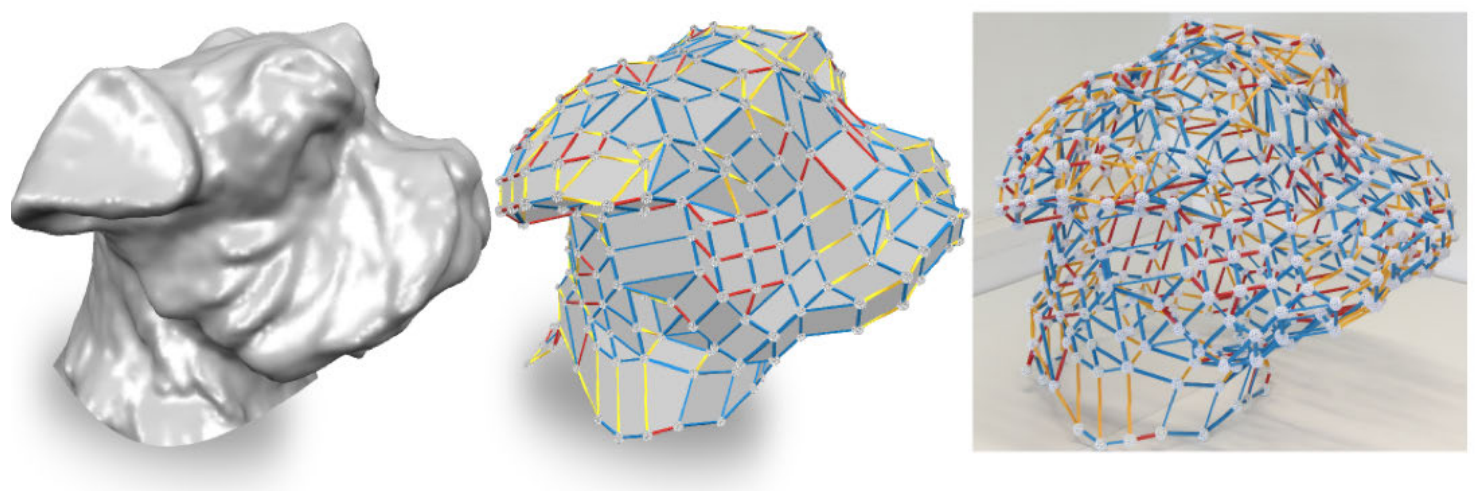

FIGURE 18. Design of a zometool obtained through the method proposed in [ZK14].

6.2. Predefined Pieces. Other researchers have investigated the possibility of creating puzzles using standard commercial piece sets, such as LEGO pieces. A method for the automatic generation of constructible models made of LEGO bricks has been proposed by Testuz et al. [TSP13]. This algorithm generates a feasible and stable LEGO configuration and the relative assembly instructions. The approach proposed in $\left[\mathrm{KLC}^{*} 15\right]$ generates pixel-art sculptures using LEGO pieces (see Figure 17, top). This approach includes a simple optimization step to improve stability and to ensure that the sculpture stands in place once assembled. Other methods start from a set of input pictures to produce a LEGO model [KTM16]. Another class of methods is more focused on how to optimize the stability of LEGO models 


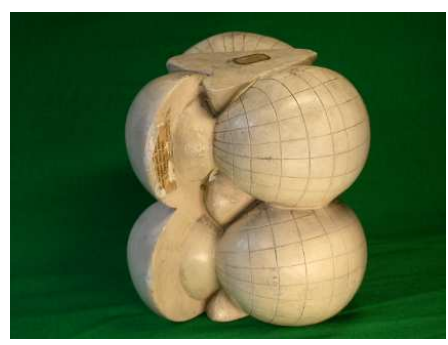

(a)

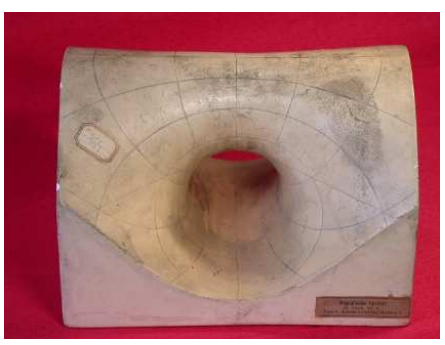

(b)

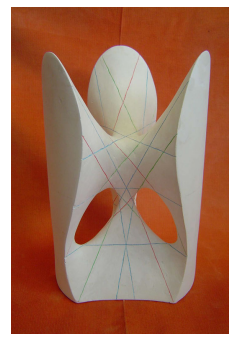

(c)

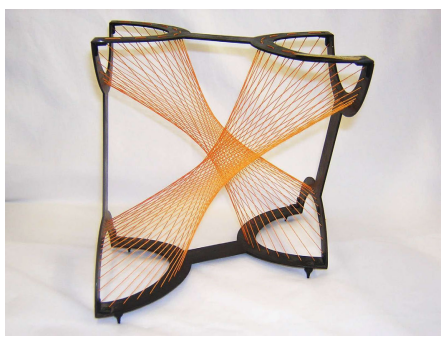

(d)

FIGURE 19. (a) Ring parabolic cyclid and (b) Sievert surface (Institute Henri Poincaré, pictures by Sabine Starita and Henri Duvillard), (c) Clebsch diagonal surface, and (d) String model representing a ruled surface with two real double lines and four real pinch points on each line (University of Groningen).

once assembled. Hong et al. [HWS*16] proposed a method to adjust the centroid of the model by changing the distribution of the pieces within the volume. More complex methods study the overall stability [WW12] or optimize the design with respect to specific physical constraints [LYH*15] (see Figure 17 , bottom).

Zometool is another method for assembling three-dimensional shapes. It is a modeling system that uses a set of different struts (three different types, each available in three different lengths) that are interconnected at nodes (one single type of node with 62 possible attaching directions). The approach proposed in [ZLAK14] finds an initial zometool approximation using a voxelization of the input mesh, and then a sequence of local operations is used to optimize the final result. A different approach based on an advancing front technique is proposed in [ZK14].

The approach proposed in [ $\left.\mathrm{KSW}^{*} 17\right]$ uses plastic bottles as basic bricks to create complex structures that are capable of supporting human weights. The bottles are connected by custom 3D printed connectors. The system supports both an interactive tool to model this kind of structure and a method to automatically convert an input shape in this kind of representation.

\section{PRinting the UnPRintable}

Actual physical shapes allow a much more natural and direct comprehension of the shape and properties of complex 3D structures. This has been exploited for didactical purpose for centuries. For example, there is a long-standing tradition in mathematics of using actual physical models, handcrafted using mixed techniques, to illustrate abstract concepts and geometrical structures [Sch03]. The University of Groningen and the Institute Henri Poincaré in Paris have large collections of mathematical objects made of gypsum, wireframes, and thread nets that are able to present complex surfaces and their properties in an accessible format [PB07]. Figure 19 shows some of these models from the two mentioned collections.

Some researchers have started to use 3D printing techniques for this purpose [KS13, Gür15], but the possibilities are still largely unexplored. It has to be noted that in the classical models, various solutions were adopted to portray specific mathematical characteristics and properties of the surfaces, such as parametric isolines, straight lines embedded in the surfaces, and singularities. The challenge of generating printable shapes that automatically collect and mark such characteristics is examined in [SS10], where the authors present a system for exploring the construction of mathematically and aesthetically interesting surfaces. Along with the use for didactic purposes, there is a connection between art and mathematical structures for artistic purposes [VC10]. Digital fabrication technologies have been used to bring abstract, mathematically defined shapes to life, with appealing results from both the aesthetic and the mathematical points of view. Also, a recent book [Seg16] is specifically targeted toward visualizing mathematical shapes using 3D printers for illustrative and educational purposes.

Manufacturing shapes that are deemed as geometrically impossible presents another interesting challenge. Prominent examples of such objects are the drawings of Escher that, intuitively, do not allow direct physical actualization. However, such figures can be built as tangible $3 \mathrm{D}$ objects by imposing constraints on the viewpoint, such as the actual creation of a Penrose triangle depicted in the sculpture of Figure 20. The creation of such structures, which seem to be in contradiction with the presented scene from at least one specific viewing direction, has been initially explored by Elber [Elb11]. In this work, he identified a class of illusions that can be realized and modeled in 3D and presented a system package that allows end users to define such seemingly impossible objects in two stages: modeling a regular 3D model and converting it into a seemingly impossible shape using special deformations. It is worth noting that there 


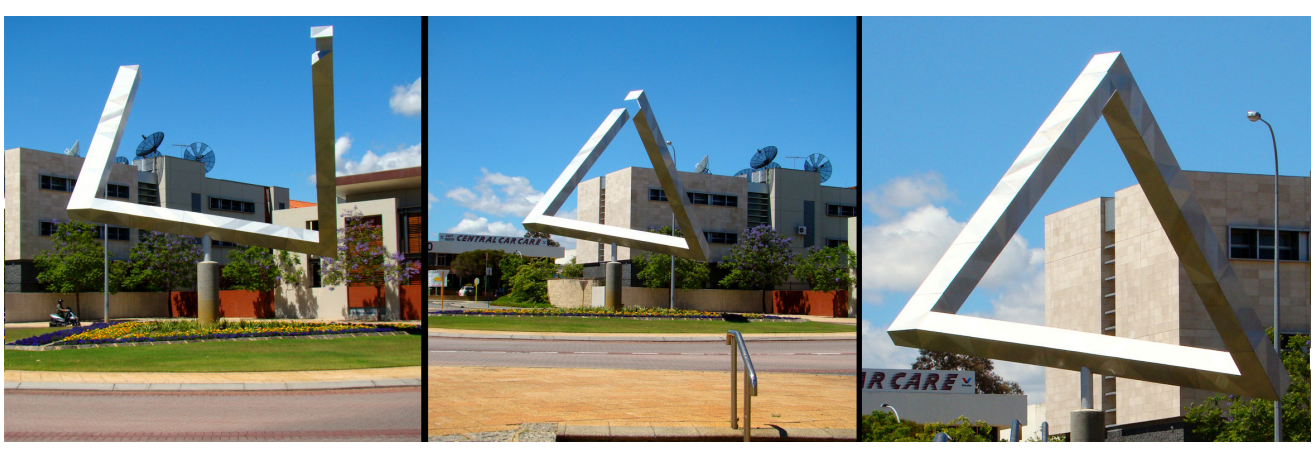

FIgURE 20. A sculpture depicting a real 3D Penrose Triangle (sculpture by Brian MacKay \& Ahmad Abas, Perth, Western Australia, photo by Bjørn Christian Tørrissen (CC BY-SA 3.0 via Wikimedia Commons).

is a connection between these approaches and the shadow art techniques [MP09]: in both cases, we have to search for shapes where the silhouettes conform to a given form, either as a shadow or as seen from a given viewpoint.

Sometimes, geometric shapes cannot be reproduced using standard digital manufacturing technologies, for example, because of the occurrence of thin features or objects' structural inadequacy. While some of these problems can be addressed by local operations such as thickening [SVB*12], we consider these methods outside of the scope of this survey. However, more challenging cases, as shown for hair [EBGB14], for example, require more sophisticated stylization approaches for 3D fabrication that are able to maintain the defining characteristics. Generalizing this method and extending it to other phenomena could be an interesting avenue for future research.

\section{OUTLOOK}

Table 1 catalogs the methods we described in this state-of-the-art report according to a number of orthogonal dimensions/characteristics. For each dimension, we give a position of that "axis" in a rough black/white domain (O/๑). We have chosen the following dimensions:

- Off the Shelf/Custom Tech: some of the presented approaches require custom specialized hardware to be implemented (O), while others use standard, commonly available fabrication technologies or ready-made components $(\bullet)$;

- Artistic/Utilitarian: most of the approaches have been conceived to solve some practical problems or to overcome the limitations of current technologies (O), while others find their primarily use as artistic expression media $(\bullet)$;

- Assembly Required/Ready-made: several fabrication techniques require manual assembly (O) compared to others for which the produced object(s) is ready once fabricated $(\bullet)$;

- Assisted Design/Blind Processing: the proposed approaches can operate with unattended processing of a given input (O), or they drive the user in the assisted design of some structure fulfilling the original purpose $(\bullet)$.

The state of the art in stylized fabrication has evolved rapidly over the last few years. Novel computational tools and output devices empower ordinary users for designing and fabricating artifacts with stunning properties. This trend can be seen as a democratization of fabrication, allowing a broader range of users to explore their creativity.

However, significant challenges remain and should be addressed in future research:

Artistic Control and User Interfaces. The design space of fabricatable artifacts is limited by the capabilities of the available output hardware and materials. Understanding these limitations and their impact on the realizable design space is highly non-trivial and often not well reflected in existing design tools. Further research is required on creative tools that allow users to navigate and explore this design space. Furthermore, when developing a design tool, a tradeoff must be made between automation and artistic control. Deeper insight into how expert designers, but also common users, interact with such tools can provide the ground for more effective and easy-to-use design interfaces.

Style Transfer. Several methods has been proposed in the literature for transferring stylistic information between 3D shapes. Style transfer can also be very useful in this domain, where the final aesthetic feeling is greatly influenced by the style of the representation. 


\begin{tabular}{|c|c|c|c|c|}
\hline Methods & $\begin{array}{l}- \text { Off the Shelf } \\
\text { O Custom Tech. }\end{array}$ & $\begin{array}{l}\text { - Artistic } \\
\text { O Utilitarian }\end{array}$ & $\begin{array}{l}\text { - Ready-made } \\
\text { O Assembly }\end{array}$ & $\begin{array}{l}\text { - Assisted } \\
\text { Design } \\
\text { O Blind } \\
\text { processing }\end{array}$ \\
\hline \multicolumn{5}{|l|}{ Developable and Foldable Patches } \\
\hline $\begin{array}{l}\text { Paper replicas [MS04b } \mid \text { STL06 } \mid \text { MGE07 } \mid \text { Tac10] } \\
\text { Curved foldings |KFC*08|KMM17] } \\
\text { Developable Strips |AKW*16] } \\
\text { WeaveMesh [TWZ*17|, Principal Strips |TISM16] } \\
\text { Origamic architectures [LSH*10. LLLN*14] } \\
\text { Multi-style pop-ups [RLYL14] }\end{array}$ & ○ & ○ & O & O \\
\hline $\begin{array}{l}\text { Origamic architectures } \text { [MS04a], V-style pop-ups } \\
\text { [LJGH11] }\end{array}$ & • & ○ & O & • \\
\hline \multicolumn{5}{|l|}{ Interlocking Flat Primitives } \\
\hline $\begin{array}{l}\text { Rigid planar pieces [MSM11, HBA12 } \text { CSaLM13, SP13. } \\
\text { CPMS14, RA15] }\end{array}$ & - & O & O & O \\
\hline FlatFitFab [MUS14], Cardboardizer [ZGPR16] & • & 0 & 0 & - \\
\hline Flexible interlocking [SCGT15 $\mid$ & - & - & O & - \\
\hline \multicolumn{5}{|l|}{ Wires and Rods } \\
\hline Wire mesh $\left.\mid \mathrm{GSFD}^{*} 14\right]$, WireDraw $\left|\mathrm{YZY}^{*} 17\right|$ & 0 & $\mathbf{0}$ & 0 & - \\
\hline Beadwork |IIM12 & ? & O & 0 & - \\
\hline Wrap jewerly |ILB15 $\mid$ & - & - & 0 & - \\
\hline Wireprint [MIG*14] & - & 0 & - & 0 \\
\hline 5dof wireprint [WPGM16 $\left|\mathrm{HZH}^{*} 16\right|$ & 0 & 0 & - & 0 \\
\hline On-the-fly print [PWMG16] & $\mathrm{O}$ & $\mathrm{O}$ & - & - \\
\hline Rod structures $\mid$ MLB16 $\mid$ & $\mathbf{0}$ & $\mathrm{O}$ & $\mathrm{O}$ & $\mathrm{O}$ \\
\hline Patterns $\left[\right.$ DLL* $^{*} 15, \mid$ MDLW15 $\left.\mid \overline{C Z X}{ }^{*} 16\right]$ & - & - & ○ & $\mathrm{O}$ \\
\hline Curve networks $\mid \overline{\mathrm{ZCT}} 16]$ & $\bullet$ & ○ & ○ & ○ \\
\hline Tensegrity structures [GCMT14 $\mid$ Tac13 PTV*17] $^{*}$ & - & 0 & 0 & 0 \\
\hline \multicolumn{5}{|l|}{ Materials } \\
\hline Garments [UKIG11], Plushie [MI07] & - & 0 & 0 & - \\
\hline Pillow $\mid$ MI06 & ○ & $\mathrm{O}$ & O & O \\
\hline Rubber balloons [STBG12], Soft objects [Hud14] & 0 & 0 & - & 0 \\
\hline Knitting compiler $\left|\mathrm{MAN}^{*} 16\right|$ & 0 & 0 & - & - \\
\hline Inflatable $\left[\mathrm{STK}^{*} 14\right]$, Printflatables [SUS*17] & 0 & 0 & 0 & - \\
\hline Kirchhoff-Plateau surfaces |POT17| & 0 & - & - & - \\
\hline CurveUps $\mid$ GMB17 $\mid$ & ○ & O & O & O \\
\hline \multicolumn{5}{|l|}{ Low-Dimensional Representations } \\
\hline Low-relief [CMS97, SRML09], Shadowpix [BBAM12] & 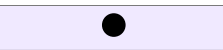 & 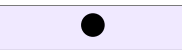 & 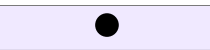 & 0 \\
\hline 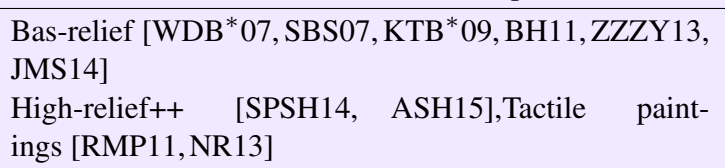 & ? & ○ & - & ○ \\
\hline \multicolumn{5}{|l|}{ Transparency, Caustics, and Shadow } \\
\hline Light routing [PRM14] & $\mathrm{O}$ & $\mathrm{O}$ & - & $\mathrm{O}$ \\
\hline Refraction pixel art [YIC $\left.{ }^{*} 12\right]$ & $\mathrm{O}$ & - & $\mathrm{O}$ & $\mathrm{O}$ \\
\hline Multilayer models [HBLM11] & 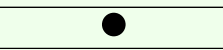 & - & O & 0 \\
\hline 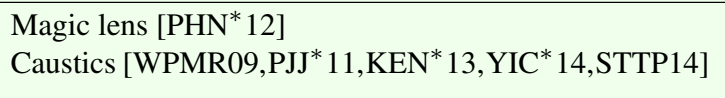 & O & - & - & O \\
\hline Shadow art [MP09| & ○ & - & - & - \\
\hline Lampshades $\left|\mathrm{ZLW}^{*} 16\right|$ & - & - & - & 0 \\
\hline \multicolumn{5}{|l|}{ Decompositions } \\
\hline $\begin{array}{l}\text { Polyomino puzzles [LFL09], Interlocking puzzles } \\
\text { [SFCO12] } \\
\text { Lego [TSP13, KTM16 } \\
\text { Zometool [ZLAK14, } \\
\text { ZKK14] }\end{array}$ & - & O & O & 0 \\
\hline Burr puzzles [XLF*11], Pixel2Brick |KLC*15] & 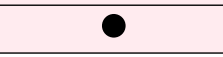 & $\mathrm{O}$ & O & $\bullet$ \\
\hline \multicolumn{5}{|l|}{ Printing the Unprintable } \\
\hline Impossible objects $|\overline{\text { Elb11 }}|$ & - & - & - & - \\
\hline Math models |KS13 Gür15| & - & 0 & - & - \\
\hline
\end{tabular}

TABLE 1. Classification of the methods reviewed in this report. 
Human Perception. Several perceptive studies have been conducted about how people perceive the appearance of photographs. A similar study should also be conducted in the field of stylized fabrication to understand the main shape characteristics that can be enhanced to beautify a fabricated model.

Prediction Accuracy. Real-word objects and the materials they are composed of can exhibit complex, nonlinear behavior. In practice, a gap is often present between the prediction of the design tool and the fabricated object. Bridging this gap can be considered one of the great challenges in stylized fabrication.

Simulation Speed. Many design interfaces require interactive simulation feedback. However, accurate simulation approaches are known to be computationally expensive. While impressive progress on fast prediction methods has been noted for various physical phenomena, such as structural stability [US13] and sound [UPSW16], the simulation cost of many others is still prohibitive for interactive applications.

Assembly Instructions. Many fabrication designs require a complex assembly sequence. These techniques can significantly benefit from a system that is capable of an intuitive visualization of the correct assembly sequence.

\section{ACKNOWLEDGMENTS}

The research leading to these results was partially funded by the EU H2020 Programme "EMOTIVE: EMOTIve Virtual cultural Experiences through personalized storytelling" H2020-SC6-CULT-COOP-08-2016 (grant agreement no. 727188), the European Research Council (ERC) under grant agreement no. 715767 "MATERIALIZABLE: Intelligent fabrication-oriented Computational Design and Modeling," and by the Italian PRIN project "DSURF" (grant no. 2015B8TRFM).

\section{REFERENCES}

[AKW*16] Akleman E., Ke S., Wu Y., Kalantar N., Borhani A., Chen J.: Construction with physical version of quad-edge data structures. Computers \& Graphics 58 (2016), 172 - 183. Shape Modeling International 2016. doi:10.1016/ j.cag.2016.05. 008

[ASH15] ARPa S., SÃusstrunk S., Hersch R. D.: High reliefs from 3d scenes. Computer Graphics Forum 34, 2 (2015), $253-263$. doi:10.1111/cgf.12557

[BBAm12] Bermano A., Baran I., Alexa M., Matusk W.: Shadowpix: Multiple images from self shadowing. Comput. Graph. Forum 31, 2pt3 (May 2012), 593-602.doi:10.1111/j.1467-8659.2012.03038.x

[BBO*10] Bickel B., Bächer M., Otaduy M. A., LeE H. R., Pfister H., Gross M., Matusik W.: Design and fabrication of materials with desired deformation behavior. ACM Trans. Graph. 29, 4 (July 2010), 63:1-63:10. doi:10.1145/1778765.1778800

[BH11] BiAn Z., HU S.-M.: Preserving detailed features in digital bas-relief making. Computer Aided Geometric Design 28, 4 (2011), 245 -256. doi:10.1016/j.cagd.2011.03.003

[BWBSH14] BÄChER M., Whiting E., Bickel B., Sorkine-Hornung O.: Spin-it: Optimizing moment of inertia for spinnable objects. ACM Trans. Graph. 33, 4 (July 2014), 96:1-96:10. doi:10.1145/2601097.2601157

[CLMK17] Chen D., Levin D. I. W., Matusik W., KAufMAn D. M.: Dynamics-aware numerical coarsening for fabrication design. ACM Trans. Graph. 36, 4 (July 2017), 84:1-84:15.doi:10.1145/3072959.3073669

[CMS97] Cignoni P., Montani C., Scopigno R.: Computer-assisted generation of bas-and high-reliefs. Journal of Graphics Tools 2 , 3 (1997), 15-28. doi:10.1080/10867651.1997.10487476

[CPMS14] Cignoni P., Pietroni N., Malomo L., Scopigno R.: Field-aligned mesh joinery. ACM Trans. Graph. 33, 1 (Feb. 2014), 11:111:12. doi: $10.1145 / 2537852$

[CSaLM13] Chen D., SitThi-AMORN P., LAN J. T., MAtusik W.: Computing and fabricating multiplanar models. Computer Graphics Forum 32, 2pt3 (2013), 305-315. doi:10.1111/cgf.12050

[CZX*16] Chen W., Zhang X., XIN S., Xia Y., LefebVRe S., WAng W.: Synthesis of filigrees for digital fabrication. ACM Trans. Graph. 35, 4 (July 2016), 98:1-98:13. doi:10.1145/2897824.2925911

[DLL*15] Dumas J., Lu A., Lefebvre S., WU J., DiCK C.: By-example synthesis of structurally sound patterns. ACM Trans. Graph. 34, 4 (July 2015), 137:1-137:12. doi:10.1145/2766984

[EBGB14] Echevarria J. I., Bradley D., Gutierrez D., Beeler T.: Capturing and stylizing hair for 3d fabrication. ACM Trans. Graph. 33, 4 (July 2014), 125:1-125:11. doi:10.1145/2601097.2601133

[Elb11] ElBer G.: Modeling (seemingly) impossible models. Computers \& Graphics 35, 3 (2011), 632 - 638. Shape Modeling International (SMI) Conference 2011. doi:10.1016/j.cag.2011.03.015

[GCMT14] Gauge D., Coros S., Mani S., Thomaszewski B.: Interactive design of modular tensegrity characters. In Proceedings of the ACM SIGGRAPH/Eurographics Symposium on Computer Animation (2014), SCA '14, Eurographics Association, pp. 131-138. doi:10.1145/2461912.2461953

[GMB17] Guseinov R., Miguel E., Bickel B.: Curveups: Shaping objects from flat plates with tension-actuated curvature. ACM Trans. Graph. 36, 4 (July 2017), 64:1-64:12. doi:10.1145/3072959.3073709

[GSFD*14] Garg A., Sageman-Furnas A. O., Deng B., Yue Y., Grinspun E., Pauly M., Wardetzky M.: Wire mesh design. ACM Trans. Graph. 33, 4 (July 2014), 66:1-66:12. doi:10.1145/2601097.2601106

[Gür15] GÜR Y.: Digital fabrication of mathematical models via low-cost 3d fdm desktop printer. Acta Physica Polonica A 128, 2B (2015). doi:10.12693/APhysPolA.128.B-100 
[HBA12] Hildebrand K., Bickel B., AleXa M.: crdbrd: Shape fabrication by sliding planar slices. Computer Graphics Forum 31, 2pt3 (2012), 583-592. doi:10.1111/j.1467-8659.2012.03037.x

[HBLM11] Holroyd M., BARAN I., LAWRence J., MatusiK W.: Computing and fabricating multilayer models. ACM Trans. Graph. 30, 6 (Dec. 2011), 187:1-187:8. doi:10.1145/2070781.2024221

[HIH*13] Hullin M. B., Ihrke I., Heidrich W., Weyrich T., Damberg G., Fuchs M.: Computational Fabrication and Display of Material Appearance. In Eurographics 2013 - State of the Art Reports (2013), Sbert M., Szirmay-Kalos L., (Eds.), The Eurographics Association.doi:10.2312/conf/EG2013/stars/137-153

[Hud14] Hudson S. E.: Printing teddy bears: A technique for 3d printing of soft interactive objects. In Proceedings of the SIGCHI Conference on Human Factors in Computing Systems (New York, NY, USA, 2014), CHI '14, ACM, pp. 459-468. doi:10.1145/2556288. 2557338

[HWS*16] Hong J.-Y., WAY D.-L., ShiH Z.-C., TAI W.-K., ChANG C.-C.: Inner engraving for the creation of a balanced lego sculpture. The Visual Computer 32, 5 (2016), 569-578. doi:10.1007/s00371-015-1072-4

[HZH* 16] Huang Y., Zhang J., Hu X., Song G., LiU Z., Yu L., LiU L.: Framefab: Robotic fabrication of frame shapes. ACM Trans. Graph. 35, 6 (Nov. 2016), 224:1-224:11.doi:10.1145/2980179.2982401

[IIM12] Igarashi Y., Igarashi T., MitAni J.: Beady: Interactive beadwork design and construction. ACM Trans. Graph. 31, 4 (July 2012), 49:1-49:9. doi:10.1145/2185520.2185545

[ILB15] IARUSSI E., Li W., Bousseau A.: Wrapit: Computer-assisted crafting of wire wrapped jewelry. ACM Trans. Graph. 34,6 (Oct. 2015), 221:1-221:8. doi:10.1145/2816795.2818118

[JMS14] Ji Z., MA W., SUN X.: Bas-relief modeling from normal images with intuitive styles. IEEE Transactions on Visualization and Computer Graphics 20, 5 (May 2014), 675-685. doi:10.1109/TVCG.2013.267

[KEN*13] Kiser T., Eigensatz M., Nguyen M. M., Bompas P., Pauly M.: Architectural Caustics - Controlling Light with Geometry. Springer Vienna, Vienna, 2013, pp. 91-106. doi:10.1007/978-3-7091-1251-9_7

[KFC*08] Kilian M., Flöry S., Chen Z., Mitra N. J., Sheffer A., Pottmann H.: Curved folding. ACM Trans. Graph. 27,3 (Aug. 2008), 75:1-75:9. doi:10.1145/1360612.1360674

[KLC*15] Kuo M.-H., Lin Y.-E., Chu H.-K., LeE R.-R., YANG Y.-L.: Pixel2brick: Constructing brick sculptures from pixel art. Comput. Graph. Forum 34, 7 (Oct. 2015), 339-348. doi:10.1111/cgf.12772

[KMM17] Kilian M., MonszPart A., Mitra N. J.: String actuated curved folded surfaces. ACM Trans. Graph. 36, 3 (May 2017), 25:125:13. doi:10.1145/3015460

[KS13] KNILl O., SLAVKOVSKY E.: Illustrating Mathematics using 3D Printers. ArXiv e-prints (June 2013). arXiv:1306.5599

[KSW*17] Kovacs R., Seufert A., Wall L., Chen H.-T., Meinel F., Müller W., You S., Brehm M., Striebel J., Kommana Y., PoPIAK A., BläsIUs T., BAUdISCH P.: Trussfab: Fabricating sturdy large-scale structures on desktop 3d printers. In Proceedings of the 2017 CHI Conference on Human Factors in Computing Systems (New York, NY, USA, 2017), CHI '17, ACM, pp. 2606-2616. doi: $10.1145 / 3025453.3026016$

[KTB*09] Kerber J., Tevs A., Belyaev A., Zayer R., Seidel H. P.: Feature sensitive bas relief generation. In 2009 IEEE International Conference on Shape Modeling and Applications (June 2009), pp. 148-154.doi:10.1109/SMI.2009.5170176

[KTM16] Kozaki T., Tedenuma H., Maekawa T.: Automatic generation of lego building instructions from multiple photographic images of real objects. Comput. Aided Des. 70, C (Jan. 2016), 13-22. doi:10.1016/ j.cad.2015.06.020

[KWC*12] Kerber J., Wang M., Chang J., Zhang J. J., Belyaev A., Seidel H.-P.: Computer assisted relief generation-a survey. Computer Graphics Forum 31, 8 (2012), 2363-2377. doi:10.1111/j.1467-8659.2012.03185.x

[LFL09] Lo K.-Y., FU C.-W., Li H.: 3d polyomino puzzle. ACM Trans. Graph. 28, 5 (Dec. 2009), 157:1-157:8. doi:10.1145/ 1618452.1618503

[LJGH11] LI X.-Y., JU T., GU Y., Hu S.-M.: A geometric study of v-style pop-ups: Theories and algorithms. ACM Trans. Graph. 30, 4 (July 2011), 98:1-98:10. doi:10.1145/2010324.1964993

[LLLN*14] LE S. N., LEOW S.-J., LE-NGUYEN T.-V., RUIZ C., LOW K.-L.: Surface and contour-preserving origamic architecture paper popups. IEEE Transactions on Visualization and Computer Graphics 20, 2 (Feb. 2014), 276-288. do i:10.1109/TVCG.2013.108

[LPRM02] LÉVy B., PETITJEAn S., RAy N., MAILlot J.: Least squares conformal maps for automatic texture atlas generation. ACM Trans. Graph. 21,3 (July 2002), 362-371. doi:10.1145/566654.566590

[LSH*10] Li X.-Y., Shen C.-H., Huang S.-S., Ju T., Hu S.-M.: Popup: Automatic paper architectures from 3d models. ACM Trans. Graph. 29, 4 (July 2010), 111:1-111:9. doi:10.1145/1778765.1778848

[LYH*15] Luo S.-J., Yue Y., HuAng C.-K., Chung Y.-H., Imai S., Nishita T., Chen B.-Y.: Legolization: Optimizing lego designs. ACM Trans. Graph. 34, 6 (Oct. 2015), 222:1-222:12. doi:10.1145/2816795.2818091

[MAN*16] McCann J., Albaugh L., Narayanan V., Grow A., Matusik W., Mankoff J., Hodgins J.: A compiler for 3d machine knitting. ACM Trans. Graph. 35, 4 (July 2016), 49:1-49:11. doi:10.1145/2897824.2925940

[MDL16] Martínez J., Dumas J., Lefebvre S.: Procedural voronoi foams for additive manufacturing. ACM Trans. Graph. 35, 4 (July 2016), 44:1-44:12. doi:10.1145/2897824.2925922

[MDLW15] Martínez J., Dumas J., LefebVRe S., Wei L.-Y.: Structure and appearance optimization for controllable shape design. ACM Trans. Graph. 34, 6 (Oct. 2015), 229:1-229:11. doi:10.1145/2816795.2818101

[MGe07] Massarwi F., Gotsman C., Elber G.: Papercraft models using generalized cylinders. In Proc. of the 15th Pacific Conf. on Computer Graphics and Applications (Washington, DC, USA, 2007), IEEE Computer Society, pp. 148-157. doi:10.1109/PG. 2007.16

[MI06] MORI Y., IgARASHI T.: Pillow: Interactive pattern design for stuffed animals. In ACM SIGGRAPH 2006 Sketches (New York, NY, USA, 2006), SIGGRAPH '06, ACM. doi:10.1145/1179849.1179942

[MI07] MORI Y., Igarashi T.: Plushie: An interactive design system for plush toys. ACM Trans. Graph. 26, 3 (July 2007). doi:10 . $1145 / 1276377.1276433$ 
[MiG*14] Mueller S., Im S., Gurevich S., Teibrich A., Pfisterer L., Guimbretière F., Baudisch P.: Wireprint: 3d printed previews for fast prototyping. In Proc. of the 27th Annual ACM Symposium on User Interface Software and Technology (New York, NY, USA, 2014), UIST '14, ACM, pp. 273-280. doi:10.1145/2642918.2647359

[MLB16] Miguel E., LePoutRE M., Bickel B.: Computational design of stable planar-rod structures. ACM Trans. Graph. 35, 4 (July 2016), 86:1-86:11.doi:10.1145/2897824.2925978

[MP09] Mitra N. J., PAUly M.: Shadow art. ACM Trans. Graph. 28, 5 (Dec. 2009), 156:1-156:7. doi:10.1145/1618452.1618502

[MS04a] MitANi J., SUZUKI H.: Computer aided design for origamic architecture models with polygonal representation. In Computer Graphics International, 2004. Proceedings (June 2004), pp. 93-99. doi:10.1109/CGI.2004.1309197.

[MS04b] Mitani J., SUZUKi H.: Making papercraft toys from meshes using strip-based approximate unfolding. ACM Trans. Graph. 23, 3 (Aug. 2004), 259-263. doi:10.1145/1015706.1015711

[MSM11] MCCrae J., Singh K., Mitra N. J.: Slices: A shape-proxy based on planar sections. ACM Trans. Graph. 30, 6 (Dec. 2011), 168:1-168:12. doi:10.1145/2070781.2024202

[MUS14] McCrae J., Umetani N., Singh K.: Flatfitfab: Interactive modeling with planar sections. In Proceedings of the 27th Annual ACM Symposium on User Interface Software and Technology (New York, NY, USA, 2014), UIST '14, ACM, pp. 13-22. doi: $10.1145 / 2642918.2647388$

[NR13] NeumÜller M., Reichinger A.: From stereoscopy to tactile photography. PhotoResearcher 19 (April 2013), 59-63.

[PB07] POLO-BLANCO I.: Theory and history of geometric models. PhD thesis, 2007.

[PHN*12] Papas M., Houit T., Nowrouzezahrai D., Gross M., Jarosz W.: The magic lens: Refractive steganography. ACM Trans. Graph. 31, 6 (Nov. 2012), 186:1-186:10. doi:10.1145/2366145.2366205

[PJJ*11] Papas M., Jarosz W., Jakob W., RusinkieWICZ S., MatusiK W., WeYRICh T.: Goal-based caustics. Computer Graphics Forum 30, 2 (2011), 503-511. doi:10.1111/j.1467-8659.2011.01876.x

[POT17] PÉREZ J., OTADUY M. A., ThOMASZEWSKi B.: Computational design and automated fabrication of kirchhoff-plateau surfaces. ACM Trans. Graph. 36, 4 (July 2017), 62:1-62:12. doi:10.1145/3072959.3073695

[PRM14] PEREIRA T., RUSinkiewiCZ S., MATUSIK W.: Computational light routing: 3d printed optical fibers for sensing and display. ACM Trans. Graph. 33, 3 (June 2014), 24:1-24:13. doi:10.1145/2602140

[PTV*17] Pietroni N., Tarini M., Vaxman A., Panozzo D., Cignoni P.: Position-based tensegrity design. ACM Trans. Graph. 36, 6 (Nov. 2017), 172:1-172:14. doi:10.1145/3130800.3130809

[PWMG16] Peng H., Wu R., Marschner S., Guimbretière F.: On-the-fly print: Incremental printing while modelling. In Proceedings of the 2016 CHI Conference on Human Factors in Computing Systems (New York, NY, USA, 2016), CHI '16, ACM, pp. 887-896. doi: $10.1145 / 2858036.2858106$

[PZM*15] Panetta J., Zhou Q., Malomo L., Pietroni N., Cignoni P., Zorin D.: Elastic textures for additive fabrication. ACM Trans. Graph. 34, 4 (July 2015), 135:1-135:12.doi:10.1145/2766937

[RA15] Richter R., AleXA M.: Beam meshes. Computers \& Graphics 53, Part A (2015), 28 - 36.40 years of Computer Graphics in Darmstadt.doi:10.1016/j.cag.2015.08.007

[RLYL14] RuIZ C. R., LE S. N., YU J., LOW K.-L.: Multi-style paper pop-up designs from 3d models. Comput. Graph. Forum 33, 2 (May 2014), 487-496. doi:10.1111/cgf.12320

[RMP11] Reichinger A., Maierhofer S., Purgathofer W.: High-quality tactile paintings. J. Comput. Cult. Herit. 4, 2 (Nov. 2011), 5:1-5:13. doi: $10.1145 / 2037820.2037822$

[Sí2] SÉQuin C. H.: Prototyping dissection puzzles with layered manufacturing. In Fabrication and Sculpture Track, Shape Modeling International Conf. (2012), Texas A\&M, May 23, 2012.

[SBR*15] Schumacher C., Bickel B., Rys J., Marschner S., Daraio C., Gross M.: Microstructures to control elasticity in 3d printing. ACM Trans. Graph. 34, 4 (July 2015), 136:1-136:13. doi:10.1145/2766926

[SBS07] Song W., Belyaev A., Seidel H.-P.: Automatic generation of bas-reliefs from 3d shapes. In Proc. of the IEEE International Conf. on Shape Modeling and Applications 2007 (Washington, DC, USA, 2007), SMI '07, IEEE Computer Society, pp. 211-214. doi:10.1109/SMI.2007.9

[SCGT15] Skouras M., Coros S., Grinspun E., Thomaszewski B.: Interactive surface design with interlocking elements. ACM Trans. Graph. 34, 6 (Oct. 2015), 224:1-224:7. doi:10.1145/2816795.2818128

[Sch03] SchIlling M.: Catalog mathematischer modelle: fur den hoheren mathematischen unterricht. Martin Schilling, 1903. URL: https://archive.org/details/catalogmathematiooschiuoft

[Seg16] Segerman H.: Visualizing Mathematics with 3D Printing. JHU Press, 2016.

[SFCO12] Song P., Fu C.-W., CohEn-OR D.: Recursive interlocking puzzles. ACM Trans. Graph. 31, 6 (Nov. 2012), 128:1-128:10. doi: $10.1145 / 2366145.2366147$

[SP12] Schwartzburg Y., Pauly M.: Design and Optimization of Orthogonally Intersecting Planar Surfaces. Springer Berlin Heidelberg, Berlin, Heidelberg, 2012, pp. 191-199. doi:10.1007/978-3-642-23435-4_22

[SP13] Schwartzburg Y., Pauly M.: Fabrication-aware design with intersecting planar pieces. Computer Graphics Forum 32, 2pt3 (2013), 317-326. doi:10.1111/cgf.12051

[SPSH14] SchÜller C., PANOZZo D., Sorkine-Hornung O.: Appearance-mimicking surfaces. ACM Trans. Graph. 33, 6 (Nov. 2014), 216:1-216:10. doi:10.1145/2661229.2661267

[SRML09] Sun X., Rosin P. L., MARTIN R. R., LANGBein F. C.: Bas-relief generation using adaptive histogram equalization. IEEE Trans on Visualization and Computer Graphics 15, 4 (July 2009), 642-653. doi:10.1109/TVCG.2009.21

[SS10] Stussak C., SCHENZEL P.: RealSurf-A Tool for the Interactive Visualization of Mathematical Models. Springer Berlin Heidelberg, Berlin, Heidelberg, 2010, pp. 173-180. doi:10.1007/978-3-642-11577-6_22

[STBG12] Skouras M., Thomaszewski B., Bickel B., Gross M.: Computational design of rubber balloons. Computer Graphics Forum 31, 2pt4 (2012), 835-844.doi:10.1111/j.1467-8659.2012.03064.x 
[STK*14] Skouras M., Thomaszewski B., Kaufmann P., Garg A., Bickel B., Grinspun E., Gross M.: Designing inflatable structures. ACM Trans. Graph. 33, 4 (July 2014), 63:1-63:10. doi:10.1145/2601097.2601166

[STL06] Shatz I., TAL A., LeIfMAn G.: Paper craft models from meshes. The Visual Computer 22, 9-11 (2006), 825-834. doi : 10 . 1007/ S00371-006-0067-6

[STTP14] Schwartzburg Y., Testuz R., Tagliasacchi A., Pauly M.: High-contrast computational caustic design. ACM Trans. Graph. 33, 4 (July 2014), 74:1-74:11. doi:10.1145/2601097.2601200

[SUS*17] SAREen H., UMAPAThi U., Shin P., KAKeHI Y., OU J., IshiI H., Maes P.: Printflatables: Printing human-scale, functional and dynamic inflatable objects. In Proceedings of the 2017 CHI Conference on Human Factors in Computing Systems (New York, NY, USA, 2017), CHI '17, ACM, pp. 3669-3680. doi:10.1145/3025453.3025898

[SVB*12] Stava O., VAneK J., Benes B., CARR N., MĚCH R.: Stress relief: Improving structural strength of 3d printable objects. ACM Trans. Graph. 31, 4 (July 2012), 48:1-48:11. doi:10.1145/2185520.2185544

[SZ15] Sun T., Zheng C.: Computational design of twisty joints and puzzles. ACM Trans. Graph. 34, 4 (July 2015), 101:1-101:11. doi:10.1145/2766961

[Tac09] TACHI T.: 3d origami design based on tucking molecule. In The Fourth International Conference on Origami in Science, Mathematics, and Education, R. Lang, ed., Pasadena (2009), pp. 259-272. doi:10.1201/b10653-27

[Tac10] TACHI T.: Origamizing polyhedral surfaces. IEEE Transactions on Visualization and Computer Graphics 16, 2 (Mar. 2010), 298-311. doi:10.1109/TVCG.2009.67.

[Tac13] TACHI T.: Interactive freeform design of tensegrity. In Advances in Architectural Geometry 2012. Springer, 2013 , pp. 259-268. doi:10.1007/978-3-7091-1251-9_21

[Tam] TAma SofTware LTD.: PePaKuRa Designer.http://www.tamasoft.co.jp/pepakura-en/

[Tib14] TiBBits S.: 4d printing: Multi-material shape change. Architectural Design 84,1 (2014), 116-121. doi:10.1002/ad.1710

[TISM16] TakeZawa M., Imai T., Shida K., MaeKawa T.: Fabrication of freeform objects by principal strips. ACM Trans. Graph. 35, 6 (Nov. 2016), 225:1-225:12. doi:10.1145/2980179.2982406

[TSP13] Testuz R., Schwartzburg Y., PAuly M.: Automatic Generation of Constructable Brick Sculptures. In Eurographics 2013 Short Papers (2013), Otaduy M.-A., Sorkine O., (Eds.), The Eurographics Association. doi:10.2312/conf/EG2013/short/ $081-084$

[TWZ*17] TAO Y., Wang G., Zhang C., Lu N., Zhang X., YaO C., Ying F.: Weavemesh: A low-fidelity and low-cost prototyping approach for 3d models created by flexible assembly. In Proceedings of the 2017 CHI Conference on Human Factors in Computing Systems (New York, NY, USA, 2017), CHI '17, ACM, pp. 509-518. doi:10.1145/3025453.3025699

[UKIG11] UMETANi N., KAUfMAn D. M., IgARAShi T., GRINSPUn E.: Sensitive couture for interactive garment modeling and editing. ACM Trans. Graph. 30, 4 (July 2011), 90:1-90:12. doi:10.1145/2010324.1964985

[UKSI14] Umetani N., Koyama Y., Schmidt R., Igarashi T.: Pteromys: Interactive design and optimization of free-formed free-flight model airplanes. ACM Trans. Graph. 33, 4 (July 2014), 65:1-65:10. doi:10.1145/2601097.2601129

[UPSW16] Umetani N., PAnotopoulou A., Schmidt R., Whiting E.: Printone: Interactive resonance simulation for free-form print-wind instrument design. ACM Trans. Graph. 35, 6 (Nov. 2016), 184:1-184:14. doi:10.1145/2980179.2980250

[US13] UMETANI N., SCHMIDT R.: Cross-sectional structural analysis for 3d printing optimization. In SIGGRAPH Asia 2013 Technical Briefs (New York, NY, USA, 2013), SA '13, ACM, pp. 5:1-5:4. doi:10.1145/2542355.2542361

[VC10] VIERLING-ClAASSEN A.: Models of surfaces and abstract art in the early 20th century. In Proceedings of Bridges 2010: Mathematics, Music, Art, Architecture, Culture (Phoenix, Arizona, 2010), Hart G. W., Sarhangi R., (Eds.), Tessellations Publishing, pp. 11-18. URL: http://archive.bridgesmathart.org/2010/bridges2010-11.html

[WDB*07] Weyrich T., Deng J., BARnes C., Rusinkiewicz S., Finkelstein A.: Digital bas-relief from $3 d$ scenes. ACM Trans. Graph. 26, 3 (July 2007). doi:10.1145/1276377.1276417

[WPGM16] Wu R., Peng H., Guimbretière F., MARsChner S.: Printing arbitrary meshes with a 5dof wireframe printer. ACM Trans. Graph. 35, 4 (July 2016), 101:1-101:9. doi:10.1145/2897824.2925966

[WPMR09] Weyrich T., PEers P., MAtUsik W., RusinkiEWICZ S.: Fabricating microgeometry for custom surface reflectance. ACM Trans. Graph. 28, 3 (July 2009), 32:1-32:6. doi:10.1145/1531326.1531338

[WW12] WASSMANn M., WEICKER K.: Maximum flow networks for stability analysis of lego@structures. In Algorithms - ESA 2012: 20th Annual European Symposium, Ljubljana, Slovenia, September 10-12, 2012. Proceedings (Berlin, Heidelberg, 2012), Epstein L., Ferragina P., (Eds.), Springer Berlin Heidelberg, pp. 813-824. doi : 10.1007/978-3-642-33090-2_70

[XLF*11] XIN S., LAI C.-F., Fu C.-W., Wong T.-T., He Y., Cohen-Or D.: Making burr puzzles from 3d models. ACM Trans. Graph. 30, 4 (July 2011), 97:1-97:8. doi:10.1145/2010324.1964992

[YCC17] YU C., CRANE K., CoROS S.: Computational design of telescoping structures. ACM Trans. Graph. 36, 4 (July 2017), 83:1-83:9. doi:10.1145/3072959.3073673

[YIC*12] Yue Y., Iwasaki K., Chen B.-Y., Dobashi Y., Nishita T.: Pixel art with refracted light by rearrangeable sticks. Computer Graphics Forum 31, 2pt3 (2012), 575-582. doi:10.1111/j.1467-8659.2012.03036.x

[YIC* 14] YUE Y., IWASAKI K., CHEN B.-Y., DOBASHI Y., NISHITA T.: Poisson-based continuous surface generation for goal-based caustics. ACM Trans. Graph. 33, 3 (June 2014), 31:1-31:7. doi:10.1145/2580946

[YZY*17] YUE Y.-T., ZhANG X., YANG Y., REN G., ChOI Y.-K., WANG W.: Wiredraw: 3d wire sculpturing guided with mixed reality. In Proceedings of the 2017 CHI Conference on Human Factors in Computing Systems (New York, NY, USA, 2017), CHI '17, ACM, pp. 3693-3704.doi:10.1145/3025453.3025792

[ZCT16] ZEHNDER J., COROS S., THOMASZEWSKI B.: Designing structurally-sound ornamental curve networks. ACM Trans. Graph. 35, 4 (July 2016), 99:1-99:10. doi:10.1145/2897824.2925888

[ZGPR16] ZhANG Y., GAO W., PAREDES L., RAMANI K.: Cardboardizer: Creatively customize, articulate and fold 3d mesh models. In Proceedings of the 2016 CHI Conference on Human Factors in Computing Systems (New York, NY, USA, 2016), CHI '16, ACM, pp. 897-907.doi:10.1145/2858036.2858362 
[ZK14] ZIMMER H., KobBELT L.: Zometool rationalization of freeform surfaces. IEEE Transactions on Visualization and Computer Graphics 20,10 (Oct 2014), 1461-1473. doi:10.1109/TVCG.2014.2307885

[ZLAK14] Zimmer H., LAFArge F., AllieZ P., Kobbelt L.: Zometool shape approximation. Graph. Models 76, 5 (Sept. 2014 ), 390-401. doi:10.1016/j.gmod.2014.03.009

[ZLW*16] Zhao H., Lu L., Wei Y., Lischinski D., Sharf A., Cohen-Or D., Chen B.: Printed perforated lampshades for continuous projective images. ACM Trans. Graph. 35, 5 (June 2016), 154:1-154:11. doi:10.1145/2907049

[ZZZY13] ZHANG Y.-W., ZHOU Y.-Q., ZhaO X.-F., YU G.: Real-time bas-relief generation from a 3d mesh. Graphical Models 75, 1 (2013), 2 -9.doi:10.1016/j.gmod.2012.10.003

\section{About the Authors}

Bend Bickel: is an Assistant Professor at the Institute of Science and Technology Austria (IST Austria), where he leads the Computer Graphics and Digital Fabrication group. Prior to this appointment, he was a Research Scientist at Disney Research Zurich and a Visiting Professor at the Technical University of Berlin. He is a computer scientist interested in computer graphics and its overlap into animation, biomechanics, material science, and digital fabrication. His main objective is to push the boundaries of how digital content can be efficiently created, simulated, and reproduced. He has published several papers on designing and reproducing the appearance and deformation behavior of objects and is a co-founder of the Symposium on Computational Fabrication.

Paolo Cignoni: is a Research Director at the Institute of Science and Information Technologies (ISTI) of the National Research Council of Italy (CNR). He received a Ph.D. degree in Computer Science at the University of Pisa in 1998. He was awarded "Best Young Researcher" by the Eurographics association in 2004. His research interests cover many Computer Graphics fields such as geometry processing, 3D scanning data processing, digital fabrication, scientific visualization, and digital heritage. He has published more than one hundred papers in international refereed journals/conferences and has served on the program committees of all the most important conferences of Computer Graphics.

Luigi Malomo: is a researcher at the Institute of Science and Information Technologies (ISTI) of the National Research Council of Italy (CNR). He received his Ph.D. degree in Computer Science at the University of Pisa. His research interests are mainly focused on computational fabrication, which he pursued during his studies, but also include geometry processing and computer human interaction.

Nico Pietroni: is a senior lecturer at the University of Technology Sydney (Australia). His research interests include geometry processing, mesh parametrization, and digital fabrication. He received a Ph.D. degree in Computer Science at the University of Genova. 